\title{
Celestial superamplitudes
}

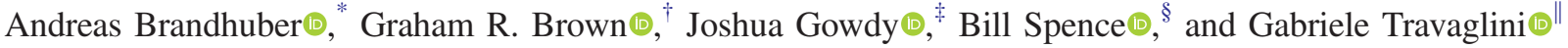 \\ Centre for Research in String Theory, School of Physics and Astronomy, \\ Queen Mary University of London, Mile End Road, London E1 4NS, United Kingdom
}

(Received 7 June 2021; accepted 29 July 2021; published 31 August 2021)

\begin{abstract}
We study celestial amplitudes in (super) Yang-Mills theory using a parametrization of the spinor helicity variables where their overall phase is not fixed by the little group action. In this approach the spin constraint $h-\bar{h}=J$ for celestial conformal primaries emerges naturally from a new Mellin transform, and the action of conformal transformations on celestial amplitudes is derived. Applying this approach to $\mathcal{N}=4$ super Yang-Mills, we show how the appropriate definition of on-shell superspace coordinates leads naturally to a formulation of chiral celestial superamplitudes and a representation of the generators of the fourdimensional superconformal algebra on the celestial sphere, which by construction annihilate all tree-level celestial superamplitudes.
\end{abstract}

DOI: 10.1103/PhysRevD.104.045016

\section{INTRODUCTION}

The quest for secret symmetries of the $S$-matrix of gauge theory and gravity has been a continuous source of surprises. An example is the discovery of the dual superconformal symmetry of $\mathcal{N}=4$ supersymmetric Yang-Mills (SYM) theory, first conjectured in [1] and subsequently proved at tree level in [2]. It emerged from earlier studies of iterative structures of maximally helicity violating (MHV) amplitudes in perturbation theory [3-5] and at strong coupling [6]. By replacing the standard kinematic variables with dual (super)momenta, a new duality of amplitudes with Wilson loops, valid at weak and strong coupling, also became apparent [6-9], with the dual symmetry acting as the usual conformal group on these new dual variables.

Recently, the work of [10-12] suggested a new way to interpret scattering amplitudes of a generic fourdimensional theory as correlators of a two-dimensional conformal field theory living at null infinity of Minkowski spacetime, also known as the celestial sphere. This builds on an earlier, important observation that the Lorentz group $\mathrm{SO}(3,1) \sim \mathrm{SL}(2, \mathbb{C}) 2 c$ of four-dimensional Minkowski space is mapped to the conformal group $\operatorname{SL}(2, \mathbb{C}) 2 c$ acting

\footnotetext{
*a.brandhuber@qmul.ac.uk

graham.brown@qmul.ac.uk

\#.k.gowdy@qmul.ac.uk

\$w.j.spence@qmul.ac.uk

g.travaglini@qmul.ac.uk
}

Published by the American Physical Society under the terms of the Creative Commons Attribution 4.0 International license. Further distribution of this work must maintain attribution to the author(s) and the published article's title, journal citation, and DOI. Funded by SCOAP. on the celestial sphere [13-15] (see [16] for a recent review). A fascinating consequence of this viewpoint is that the well-known soft theorems of gauge theories and gravity appear as Ward identities in the celestial conformal field theory [17-27]. In fact, the natural group acting on the sphere at null infinity is the infinite-dimensional Bondi-van der Burg-Metzner-Sachs group [28,29], which is expected to be a symmetry of the dual celestial conformal field theory.

The mapping from Minkowski space to the celestial sphere occurs through a Mellin transform which maps momentum eigenstates to boost eigenstates-conformal wavepackets with well-defined conformal weights. The special features of this new basis have been discussed in [10-12,30-32], in particular the boost eigenstates have unusual properties such as the nondecoupling of infrared and ultraviolet physics, as emphasized in [32], which also gives a new angle on a possible nonperturbative $S$-matrix bootstrap by looking at the analytic properties of the celestial $S$-matrix. We also note the recent works of $[33,34]$, which study the universal infrared divergences of celestial amplitudes in Yang-Mills theory, building on earlier work on gravity and quantum electrodynamics $[32,35]$. Most of the attention so far has been on tree amplitudes, with the exceptions of [33,34,36,37], which discussed the iterative structure of loop amplitudes or infrared divergences thereof.

Work on supersymmetric theories has been mostly restricted to minimal $\mathcal{N}=1$ supersymmetric models [27,38], whereas in this paper we focus on the maximally supersymmetric $\mathcal{N}=4$ theory, its $S$-matrix and symmetries. One of the questions we will answer is how to systematically derive the action of the full superconformal group on celestial (super)amplitudes, extending the work of [39] which presented the form of the generators of the 
four-dimensional conformal group on the celestial sphere. The Lorentz subgroup is of course implemented trivially as two-dimensional conformal transformations, however the remaining generators give rise to highly nontrivial constraints on a yet to be found celestial conformal field theory. The question then naturally arises as to what the celestial operators corresponding to the generators of the full four-dimensional superconformal group are.

In order to answer this question in a systematic way, in particular ensuring that the new generators in celestial space naturally obey the superconformal algebra, we find it useful to introduce a new "chiral" Mellin transform. In this approach we define the Mellin transform to celestial space in terms of standard spinor helicity variables without modding out the little group redundancy occurring in the expression of a null momentum in terms of spinor variables. This has the advantage of making the translation between Minkowski space generators and celestial generators immediate. Using our new Mellin transform we define corresponding celestial states and (super)amplitudes (in the supersymmetric case). After performing the integration over the redundant little group phase, our celestial states and amplitudes are equal to the conventional ones multiplied by a delta function imposing the spin constraint $h-$ $\bar{h}=J$ much in the same way as the momentum-conserving delta function in Minkowski spacetime. This allows us to give an unambiguous definition of the action of celestial operators and of their weights. In particular we discuss extensively how to compute weights of all the relevant operators in celestial space. We also present explicit expressions for tree-level MHV superamplitudes. Our results here agree with those of [40], although the specific forms of the superamplitudes we present are new.

The rest of the paper is organized as follows. In Sec. II we review the usual nonchiral Mellin transform and introduce our chiral celestial coordinates along with their transformations under the Lorentz group. We also discuss the relation between these two sets of coordinates. In Sec. III we introduce chiral celestial states and amplitudes, and discuss the action of the Lorentz group on these quantities. Section IV is devoted to the derivation of the generators of the conformal group in Mellin space, showing the usefulness of our chiral representation. In this section we also explain how to determine the weights of operators in celestial space. After briefly reviewing basic properties of superamplitudes in $\mathcal{N}=4 \mathrm{SYM}$, we present in Sec. V the formal derivation of celestial superamplitudes in this theory, together with explicit examples at three, four and five points, and comments about the general $n$-point case. Section VI is devoted to the derivation of explicit expressions for the full set of generators of the $\mathcal{N}=4$ superconformal algebra. Finally, in Sec. VII we present our conclusions and outline possible lines of further research. The Appendix expands on the derivation of weights of celestial operators, and in particular we present in Table I the list of weights of all relevant celestial operators.
TABLE I. Table of conformal weights for objects defined or acting in Mellin space.

Table of conformal weights

\begin{tabular}{|c|c|c|}
\hline Celestial object & $(h, \bar{h})$ & $(\Delta, J)$ \\
\hline$\tilde{A}_{J}$ & $\left(\frac{\Delta+J}{2}, \frac{\Delta-J}{2}\right)$ & $(\Delta, J)$ \\
\hline$\lambda_{\alpha}$ & $\left(-\frac{1}{4}, \frac{1}{4}\right)$ & $\left(0,-\frac{1}{2}\right)$ \\
\hline$\tilde{\lambda}_{\dot{\alpha}}$ & $\left(\frac{1}{4},-\frac{1}{4}\right)$ & $\left(0, \frac{1}{2}\right)$ \\
\hline$\partial_{\alpha}$ & $\left(\frac{1}{4},-\frac{1}{4}\right)$ & $\left(0, \frac{1}{2}\right)$ \\
\hline$\tilde{\partial}_{\dot{\alpha}}$ & $\left(-\frac{1}{4}, \frac{1}{4}\right)$ & $\left(0,-\frac{1}{2}\right)$ \\
\hline$\eta^{A}$ & $\left(\frac{1}{4},-\frac{1}{4}\right)$ & $\left(0, \frac{1}{2}\right)$ \\
\hline$\partial_{A}$ & $\left(-\frac{1}{4}, \frac{1}{4}\right)$ & $\left(0,-\frac{1}{2}\right)$ \\
\hline$\xi_{\alpha}=(z, 1)_{\alpha}$ & $\left(-\frac{1}{2}, 0\right)$ & $\left(-\frac{1}{2},-\frac{1}{2}\right)$ \\
\hline$\tilde{\xi}_{\dot{\alpha}}=(\bar{z}, 1)_{\dot{\alpha}}$ & $\left(0,-\frac{1}{2}\right)$ & $\left(-\frac{1}{2}, \frac{1}{2}\right)$ \\
\hline$\left(\begin{array}{c}-2\left(h-\frac{1}{4} \tau^{A} \check{\partial}_{A}\right)+1-z \partial \\
-\partial\end{array}\right)_{\alpha}$ & $\left(\frac{1}{2}, 0\right)$ & $\left(\frac{1}{2}, \frac{1}{2}\right)$ \\
\hline$\left(\begin{array}{c}-2\left(\bar{h}+\frac{1}{4} \tau^{A} \check{\partial}_{A}\right)+1-\bar{z} \bar{\partial} \\
-\bar{\partial}\end{array}\right)$. & $\left(0, \frac{1}{2}\right)$ & $\left(\frac{1}{2},-\frac{1}{2}\right)$ \\
\hline$\tau^{A}$ & $\left(\frac{1}{4},-\frac{1}{4}\right)$ & $\left(0, \frac{1}{2}\right)$ \\
\hline$\check{\partial}_{A}$ & $\left(-\frac{1}{4}, \frac{1}{4}\right)$ & $\left(0,-\frac{1}{2}\right)$ \\
\hline$e^{\frac{\partial h}{2}}$ & $\left(\frac{1}{4}, \frac{1}{4}\right)$ & $\left(\frac{1}{2}, 0\right)$ \\
\hline$e^{\frac{\partial_{\bar{h}}}{2}}$ & $\left(\frac{1}{4}, \frac{1}{4}\right)$ & $\left(\frac{1}{2}, 0\right)$ \\
\hline$e^{-\frac{\partial_{h}}{4}+\frac{\partial_{\bar{h}}}{4}}$ & $(0,0)$ & $(0,0)$ \\
\hline
\end{tabular}

\section{CELESTIAL COORDINATES}

\section{A. Review of nonchiral celestial coordinates}

As usual in four spacetime dimensions, we write massless momenta in terms of spinor helicity variables as $p_{\alpha \dot{\alpha}}=\lambda_{\alpha} \tilde{\lambda}_{\dot{\alpha}}$. Little group transformations,

$$
\lambda_{\alpha} \rightarrow e^{i \phi} \lambda_{\alpha}, \quad \tilde{\lambda}_{\dot{\alpha}} \rightarrow e^{-i \phi} \tilde{\lambda}_{\dot{\alpha}},
$$

leave a momentum invariant, where we are restricting to the Minkowski slice where $p^{\mu}$ is real and $\tilde{\lambda}_{\dot{\alpha}}=\epsilon \lambda_{\alpha}^{*}$, where $\epsilon$ is an overall sign which is positive (negative) for outgoing (incoming) momenta. Out of the four real degrees of freedom in $\lambda_{\alpha}$, three are physical while its overall phase $e^{i \theta}$ is redundant. It can be set to zero by a little group transformation with $\phi=-\theta$.

Transitioning from the usual language of scattering amplitudes in four-dimensional Minkowski spacetime to conformal correlators on the celestial sphere $\mathcal{C S}$ requires one to introduce new coordinates [10-12,20,23,24,30,31,39],

$$
p_{\alpha \dot{\alpha}}:=\epsilon \omega q_{\alpha \dot{\alpha}},
$$

where $q_{\alpha \dot{\alpha}}:=\xi_{\alpha} \tilde{\xi}_{\dot{\alpha}}$, with ${ }^{1}$

\footnotetext{
${ }^{1}$ We omit explicit spinor indices when there is no ambiguity.
} 


$$
\xi_{\alpha}=\left(\begin{array}{c}
z \\
1
\end{array}\right), \quad \tilde{\xi}_{\dot{\alpha}}=\left(\begin{array}{c}
\bar{z} \\
1
\end{array}\right)
$$

In vector notation,

$$
q^{\mu}:=\frac{1}{2}\left(1+|z|^{2},-z-\bar{z},-i(z-\bar{z}), 1-|z|^{2}\right) .
$$

The energy of the particle is $p^{0}=\frac{\omega}{2}\left(1+|z|^{2}\right)$, however, following standard convention we will refer to $\omega$ as the "energy."

Following [32], one can parametrize spinors in terms of the physical degrees of freedom:

$$
\lambda_{\alpha}=\epsilon \sqrt{\omega}\left(\begin{array}{c}
z \\
1
\end{array}\right), \quad \tilde{\lambda}_{\dot{\alpha}}=\sqrt{\omega}\left(\begin{array}{l}
\bar{z} \\
1
\end{array}\right)
$$

where a little group transformation was used to set $\lambda_{2}=$ $\sqrt{\omega}$ to be real. The spinors $\lambda_{\alpha}$ and $\tilde{\lambda}_{\dot{\alpha}}$ naturally transform in the (anti)fundamental representation of $\operatorname{SL}(2, \mathbb{C}) 2 c$ :

$$
\lambda \rightarrow \lambda^{\prime}=\left(\begin{array}{ll}
a & b \\
c & d
\end{array}\right) \lambda, \quad \tilde{\lambda} \rightarrow \tilde{\lambda}^{\prime}=\left(\begin{array}{cc}
\bar{a} & \bar{b} \\
\bar{c} & \bar{d}
\end{array}\right) \tilde{\lambda}
$$

Hence, under a Lorentz transformation $p_{\alpha \dot{\alpha}}$ transforms as

$$
\begin{aligned}
p_{\alpha \dot{\alpha}} & \rightarrow \epsilon \omega(c z+d)(\bar{c} \bar{z}+\bar{d})\left(\begin{array}{c}
\frac{a z+b}{c z+d} \\
1
\end{array}\right)\left(\begin{array}{c}
\frac{\bar{a} \bar{z}+\bar{b}}{\bar{c} \bar{z}+\bar{d}} \\
1
\end{array}\right) \\
& =\epsilon \omega^{\prime}\left(\begin{array}{c}
z^{\prime} \\
1
\end{array}\right)\left(\begin{array}{c}
\bar{z}^{\prime} \\
1
\end{array}\right)=\epsilon \omega^{\prime}\left(\begin{array}{cc}
z^{\prime} \bar{z}^{\prime} & z^{\prime} \\
\bar{z}^{\prime} & 1
\end{array}\right),
\end{aligned}
$$

where

$z^{\prime}:=\frac{a z+b}{c z+d}, \quad \bar{z}^{\prime}:=\frac{\bar{a} \bar{z}+\bar{b}}{\bar{c} \bar{z}+\bar{d}}, \quad a d-b c=1$,

which is a Möbius transformation on $\mathcal{C S}^{2}$, and

$$
\omega \rightarrow \omega^{\prime}=(c z+d)(\bar{c} \bar{z}+\bar{d}) \omega
$$

In the $(\omega, z, \bar{z})$ parametrization we can then write Lorentz transformations on the spinors $\lambda_{\alpha}$ and $\tilde{\lambda}_{\dot{\alpha}}$ as [32]

$$
\begin{aligned}
& \lambda \rightarrow \lambda^{\prime}=\sqrt{\omega}\left(\begin{array}{ll}
a & b \\
c & d
\end{array}\right)\left(\begin{array}{l}
z \\
1
\end{array}\right)=\left(\frac{\bar{c} \bar{z}+\bar{d}}{c z+d}\right)^{-1 / 2} \sqrt{\omega^{\prime}}\left(\begin{array}{c}
z^{\prime} \\
1
\end{array}\right), \\
& \tilde{\lambda} \rightarrow \tilde{\lambda}^{\prime}=\sqrt{\omega}\left(\begin{array}{ll}
\bar{a} & \bar{b} \\
\bar{c} & \bar{d}
\end{array}\right)\left(\begin{array}{l}
\bar{z} \\
1
\end{array}\right)=\left(\frac{\bar{c} \bar{z}+\bar{d}}{c z+d}\right)^{1 / 2} \sqrt{\omega^{\prime}}\left(\begin{array}{c}
\bar{z}^{\prime} \\
1
\end{array}\right) .
\end{aligned}
$$

Note that just transforming the coordinates $\omega \rightarrow \omega^{\prime}$ and $z \rightarrow z^{\prime}$ is not the same as performing a Lorentz transformation due to the additional phase factors in (2.10), which appear as a consequence of the particular parametrization chosen. Finally we note the action of a Lorentz transformation on $q$,

$$
q^{\mu} \rightarrow \Lambda_{\nu}^{\mu} q^{\nu}=(c z+d)(\bar{c} \bar{z}+\bar{d}) q^{\prime \mu}
$$

where $q_{\alpha \dot{\alpha}}^{\prime}=\xi_{\alpha}^{\prime} \tilde{\xi}_{\dot{\alpha}}^{\prime}$, with $\xi_{\alpha}^{\prime}=\left(\begin{array}{c}z^{\prime} \\ 1\end{array}\right)$ and $\tilde{\xi}_{\dot{\alpha}}^{\prime}=\left(\begin{array}{c}\bar{z}^{\prime} \\ 1\end{array}\right)$.

\section{B. Chiral celestial coordinates}

In this paper we will employ a different parametrization of the spinors which does not fix the little group redundancy. This has a number of advantages as we will see in the following. In this parametrization, we define

$\lambda_{\alpha}:=\epsilon u\left(\begin{array}{c}z \\ 1\end{array}\right)=\epsilon u \xi_{\alpha}, \quad \tilde{\lambda}_{\dot{\alpha}}:=\bar{u}\left(\begin{array}{l}\bar{z} \\ 1\end{array}\right)=\bar{u} \tilde{\xi}_{\dot{\alpha}}$

where $u$ is a complex number such that

$$
u \bar{u}=\omega
$$

It is immediate to relate this "chiral" parametrization of the spinors to the "nonchiral" one in (2.5). Setting

$$
u=\sqrt{\omega} e^{i \theta}, \quad \bar{u}=\sqrt{\omega} e^{-i \theta},
$$

the connection between the two is simply

$$
\lambda_{\mathrm{C}}=e^{i \theta} \lambda_{\mathrm{NC}}, \quad \tilde{\lambda}_{\mathrm{C}}=e^{-i \theta} \tilde{\lambda}_{\mathrm{NC}},
$$

with $e^{i \theta}=\sqrt{u / \bar{u}}$. Thus the parametrization (2.12) contains an additional degree of freedom. In the following we will regard an amplitude as a function of the set of variables $\{z, \bar{z}, u, \bar{u}\}$. We will call these coordinates chiral celestial coordinates or just chiral coordinates, as opposed to the original nonchiral celestial coordinates $\{z, \bar{z}, \omega\}$. Unlike in the nonchiral case, the chiral coordinates are a straightforward change of variables from the original spinor components $\lambda_{1}, \lambda_{2}, \tilde{\lambda}_{i}, \tilde{\lambda}_{\dot{2}}$. Furthermore, in the chiral parametrization (2.12), a Lorentz transformation of the spinors $\lambda_{\alpha}$ and $\tilde{\lambda}_{\dot{\alpha}}$ is written as a standard $\operatorname{SL}(2, \mathbb{C}) 2 c$ transformation,

$$
\begin{aligned}
& \lambda \rightarrow \lambda^{\prime}=u\left(\begin{array}{ll}
a & b \\
c & d
\end{array}\right)\left(\begin{array}{l}
z \\
1
\end{array}\right)=u^{\prime}\left(\begin{array}{l}
z^{\prime} \\
1
\end{array}\right), \\
& \tilde{\lambda} \rightarrow \tilde{\lambda}^{\prime}=\bar{u}\left(\begin{array}{ll}
\bar{a} & \bar{b} \\
\bar{c} & \bar{d}
\end{array}\right)\left(\begin{array}{l}
\bar{z} \\
1
\end{array}\right)=\bar{u}^{\prime}\left(\begin{array}{l}
\bar{z}^{\prime} \\
1
\end{array}\right),
\end{aligned}
$$

where

$$
u \rightarrow u^{\prime}=(c z+d) u, \quad \bar{u} \rightarrow \bar{u}^{\prime}=(\bar{c} \bar{z}+\bar{d}) \bar{u} .
$$

It is instructive to contrast (2.16) with (2.10) — in the former the Lorentz transformation is equivalent to a Möbius 
coordinate transformation, while in the latter an additional phase factor is needed.

\section{STATES AND AMPLITUDES IN MELLIN SPACE}

In momentum space, consider the scattering of asymptotic states labeled by their momentum $p^{\mu}$ and helicity $J$, where, under a little group transformation (2.1),

$$
\left|p^{\mu} ; J\right\rangle \rightarrow\left|p^{\mu} ; J\right\rangle^{\prime}=\left(e^{i \phi}\right)^{-2 J}\left|p^{\mu} ; J\right\rangle .
$$

We can alternatively label states using spinor variables as $\left|\lambda_{\alpha}, \tilde{\lambda}_{\dot{\alpha}} ; J\right\rangle$. Then, in the chiral and nonchiral celestial coordinates we write

$$
\text { Chiral: }|z, \bar{z}, u, \bar{u} ; J\rangle:=\left|u \xi_{\alpha}, \bar{u} \bar{\xi}_{\dot{\alpha}} ; J\right\rangle \text {, }
$$

Nonchiral: $|z, \bar{z}, \omega ; J\rangle:=\left|\sqrt{\omega} \xi_{\alpha}, \sqrt{\omega} \bar{\xi}_{\dot{\alpha}} ; J\right\rangle$.

The two states are just related by the little group transformation in (2.15),

$$
|z, \bar{z}, u, \bar{u} ; J\rangle=\left(e^{i \theta}\right)^{-2 J}|z, \bar{z}, \omega ; J\rangle,
$$

with $e^{i \theta}=\sqrt{u / \bar{u}}$. This extends immediately to a relation between amplitudes written in terms of chiral and nonchiral coordinates:

$A\left(\left\{z_{i}, \bar{z}_{i}, u_{i}, \bar{u}_{i} ; J_{i}\right\}\right)=\prod_{j=1}^{n}\left(e^{i \theta_{j}}\right)^{-2 J_{j}} A\left(\left\{z_{i}, \bar{z}_{i}, \omega_{i} ; J_{i}\right\}\right)$,

with $e^{i \theta_{j}}=\sqrt{u_{j} / \bar{u}_{j}}$.

\section{A. Nonchiral Mellin transform}

In the nonchiral approach of [10-12] one defines conformal primary states using a Mellin transform with respect to the energy of the particle,

$$
|z, \bar{z}, \Delta ; J\rangle:=\int_{0}^{\infty} d \omega \omega^{\Delta-1}|z, \bar{z}, \omega ; J\rangle,
$$

where $\Delta$ is the two-dimensional conformal weight of the particle. Importantly, this state transforms as a conformal primary under the $\operatorname{SL}(2, \mathbb{C})$ (or equivalently the Lorentz) symmetry of the celestial sphere. From (2.10) it follows that, under a Lorentz transformation,

$$
|z, \bar{z}, \omega ; J\rangle \rightarrow\left|z^{\prime}, \bar{z}^{\prime}, \omega^{\prime} ; J\right\rangle\left(\frac{c z+d}{\bar{c} \bar{z}+\bar{d}}\right)^{-J},
$$

hence, using (3.5) and (2.9),

$$
\begin{aligned}
|z, \bar{z}, \Delta ; J\rangle & \rightarrow(c z+d)^{-\Delta-J}(\bar{c} \bar{z}+\bar{d})^{-\Delta+J}\left|z^{\prime}, \bar{z}^{\prime}, \Delta ; J\right\rangle \\
& =\left(\frac{\partial z^{\prime}}{\partial z}\right)^{h}\left(\frac{\partial \bar{z}^{\prime}}{\partial \bar{z}}\right)^{\bar{h}}\left|z^{\prime}, \bar{z}^{\prime}, \Delta ; J\right\rangle,
\end{aligned}
$$

which is the transformation law for a two-dimensional conformal primary with weights given in terms of $\Delta, J$ by

$$
h=\frac{\Delta+J}{2}, \quad \bar{h}=\frac{\Delta-J}{2} .
$$

Hence these conformal primary states can be labeled by $\{h, \bar{h}\}$ or $\{\Delta, J\}[10]$.

\section{B. Chiral Mellin transform}

With the chiral variables $u, \bar{u}$ we now define a new Mellin transform as

$|z, \bar{z}, h, \bar{h} ; J\rangle:=\frac{1}{2 \pi i} \int_{\mathbb{C}} d u \wedge d \bar{u} u^{2 h-1} \bar{u}^{2 \bar{h}-1}|z, \bar{z}, u, \bar{u} ; J\rangle$.

This is natural, since changing integration variables from $u, \bar{u}$ to $\omega, \theta$ using (2.14), and recalling (3.3), we find

$$
\begin{aligned}
|z, \bar{z}, h, \bar{h} ; J\rangle= & \frac{1}{2 \pi} \int_{0}^{2 \pi} d \theta\left(e^{2 i \theta}\right)^{(h-\bar{h}-J)} \\
& \times \int_{0}^{\infty} d \omega \omega^{h+\bar{h}-1}|z, \bar{z}, \omega ; J\rangle .
\end{aligned}
$$

Since we wish to consider conformal primaries with the usual half-integer helicity, we restrict $(h-\bar{h}) \in \frac{1}{2} \mathbb{Z}$, and we arrive at

$|z, \bar{z}, h, \bar{h} ; J\rangle=\delta_{h-\bar{h}-J, 0} \int_{0}^{\infty} d \omega \omega^{h+\bar{h}-1}|z, \bar{z}, \omega ; J\rangle$.

Thus the state vanishes when $h-\bar{h} \neq J$, while when $h-\bar{h}=J$ it coincides with the nonchiral conformal primary state.

In the nonchiral case, a celestial amplitude is not initially written as a function of $h$ and $\bar{h}$, since these quantities do not appear explicitly in the $\omega$ Mellin integral; in order to write it as such we must substitute according to the condition $h-\bar{h}=J$ by hand. In contrast, in the chiral Mellin transform $h$ and $\bar{h}$ are introduced a priori as free parameters varying within the constraint $(h-\bar{h}) \in \frac{1}{2} \mathbb{Z}$; the chiral conformal primary is manifestly a function of $h, \bar{h}$ which, however, is only nonvanishing when $h-\bar{h}=J$. The natural appearance of the spin constraint means that states have the expected weights $(h, \bar{h})=\left(\frac{\Delta+J}{2}, \frac{\Delta-J}{2}\right)$ selected by their helicity. The fact that $h$ and $\bar{h}$ are now explicit in the chiral Mellin transform also makes the action of operators which shift conformal weight, such as $e^{\partial_{h} / 2}$, unambiguous, as discussed in Sec. IV B. 
Finally we note that under a Lorentz transformation

$$
|z, \bar{z}, u, \bar{u} ; J\rangle \rightarrow\left|z^{\prime}, \bar{z}^{\prime}, u^{\prime}, \bar{u}^{\prime} ; J\right\rangle
$$

which follows simply from (2.16).

\section{Celestial amplitudes: chiral vs nonchiral}

The discussion of chiral and nonchiral states extends naturally to amplitudes as follows:

$$
\begin{aligned}
& \text { Chiral: } \tilde{A}^{\mathrm{C}}\left(\left\{z_{i}, \bar{z}_{i}, h_{i}, \bar{h}_{i}, J_{i}\right\}\right):=\frac{1}{(2 \pi i)^{n}} \prod_{j=1}^{n} \int_{\mathbb{C}} d u_{j} \wedge d \bar{u}_{j} u^{2 h_{j}-1} \bar{u}^{2 \bar{h}_{j}-1} A\left(\left\{z_{i}, \bar{z}_{i}, u_{i}, \bar{u}_{i}, J_{i}\right\}\right), \\
& \text { Nonchiral: } \quad \tilde{A}^{\mathrm{NC}}\left(\left\{z_{i}, \bar{z}_{i}, \Delta_{i}, J_{i}\right\}\right):=\prod_{j=1}^{n} \int_{0}^{\infty} d \omega_{j} \omega_{j}^{\Delta-1} A\left(\left\{z_{i}, \bar{z}_{i}, \omega_{i}, J_{i}\right\}\right) .
\end{aligned}
$$

We can compare these in a similar way to what was done for the states. We change variables from $(u, \bar{u})$ to $(\omega, \theta)$ coordinates, and noting (3.4) we arrive at

$$
\begin{aligned}
\tilde{A}^{\mathrm{C}}\left(\left\{z_{i}, \bar{z}_{i}, h_{i}, \bar{h}_{i}, J_{i}\right\}\right) & =\prod_{j=1}^{n} \delta_{h_{j}-\bar{h}_{j}-J_{j}, 0} \int_{0}^{\infty} d \omega_{j} \omega_{j}^{h_{j}+\bar{h}_{j}-1} A\left(\left\{z_{i}, \bar{z}_{i}, \omega_{i}, J_{i}\right\}\right) \\
& =\prod_{j=1}^{n} \delta_{h_{j}-\bar{h}_{j}-J_{j}, 0} \tilde{A}^{\mathrm{NC}}\left(\left\{z_{i}, \bar{z}_{i}, \Delta_{i}, J_{i}\right\}\right) .
\end{aligned}
$$

We conclude that the nonchiral celestial amplitude is simply the chiral celestial amplitude with the Kronecker deltas $\delta_{h-\bar{h}-J, 0}$ stripped off for each particle. This is analogous to the situation where we strip off the momentum-conserving delta function from an amplitude and impose momentum conservation "by hand" instead. For the rest of this paper we will work with chiral celestial amplitudes (3.13a) and use the representation (3.13b) when needed. ${ }^{2}$

It is also instructive to consider the Lorentz transformation of a chiral conformal primary state (3.9). Recalling (3.12), we have

$$
\begin{aligned}
|z, \bar{z}, h, \bar{h} ; J\rangle & \rightarrow \frac{1}{2 \pi i} \int_{\mathbb{C}} d u \wedge d \bar{u} u^{2 h-1} \bar{u}^{2 \bar{h}-1}\left|z^{\prime}, \bar{z}^{\prime}, u^{\prime}, \bar{u}^{\prime} ; J\right\rangle \\
& =(c z+d)^{-2 h}(\bar{c} \bar{z}+\bar{d})^{-2 \bar{h}}\left|z^{\prime}, \bar{z}^{\prime}, h, \bar{h} ; J\right\rangle .
\end{aligned}
$$

Thus the state transforms under the two-dimensional conformal group with weights given by the parameters $h$ and $\bar{h}$ appearing in the chiral Mellin transform. These considerations extend to amplitudes - using the invariance of an amplitude under Lorentz transformations and (3.15) we have

$$
\tilde{A}\left(\left\{z_{i}, \bar{z}_{i}, h_{i}, \bar{h}_{i}, J_{i}\right\}\right)=\prod_{j=1}^{n}\left(c z_{j}+d\right)^{-2 h_{j}}\left(\bar{c} \bar{z}_{j}+\bar{d}\right)^{-2 \bar{h}_{j}} \tilde{A}\left(\left\{z_{i}^{\prime}, \bar{z}_{i}^{\prime}, h_{i}, \bar{h}_{i}, J_{i}\right\}\right),
$$

which is just the transformation for a correlator of $n$ two-dimensional conformal primaries, as expected.

\section{FROM CHIRAL COORDINATES TO THE CELESTIAL CONFORMAL ALGEBRA}

As a first demonstration of the use of chiral coordinates and the chiral Mellin transform, we rederive the celestial representation of the four-dimensional conformal generators first presented in [39].

\footnotetext{
${ }^{2}$ From now on we will also drop the superscripts $\mathrm{C}$ and $\mathrm{NC}$ in the celestial amplitudes, unless we wish to make the distinction clear.
} 


\section{A. Celestial conformal generators}

Our first goal is to find representations of $\lambda_{\alpha}$ and $\tilde{\lambda}_{\dot{\alpha}}$ on the celestial sphere, that is derive their induced action on celestial amplitudes in Mellin space. In chiral coordinates this is straightforward. First, we change variables from $\left\{\lambda_{\alpha}, \tilde{\lambda}_{\dot{\alpha}}\right\}$ to $\{z, \bar{z}, u, \bar{u}\}$, with

$$
\begin{aligned}
& \lambda_{\alpha}=u\left(\begin{array}{c}
z \\
1
\end{array}\right), \quad \tilde{\lambda}_{\dot{\alpha}}=\bar{u}\left(\begin{array}{c}
\bar{z} \\
1
\end{array}\right), \\
& \partial_{\alpha}:=\frac{\partial}{\partial \lambda^{\alpha}}=\frac{1}{u}\left(\begin{array}{c}
u \partial_{u}-z \partial \\
-\partial
\end{array}\right), \quad \tilde{\partial}_{\dot{\alpha}}:=\frac{\partial}{\partial \tilde{\lambda}^{\dot{\alpha}}}=\frac{1}{\bar{u}}\left(\begin{array}{c}
\bar{u} \partial_{\bar{u}}-\bar{z} \bar{\partial} \\
-\bar{\partial}
\end{array}\right),
\end{aligned}
$$

where $\partial_{u}=\partial / \partial u$ and $\partial_{\bar{u}}=\partial / \partial \bar{u}$. Next we define the action of these objects on celestial amplitudes using the chiral Mellin transform. For instance, for $\lambda_{\alpha}$

$$
\lambda_{\alpha} \tilde{A}=\frac{1}{2 \pi i} \int_{\mathbb{C}} d u \wedge d \bar{u} u^{2 h-1} \bar{u}^{2 \bar{h}-1} u\left(\begin{array}{l}
z \\
1
\end{array}\right) A=\left(\begin{array}{l}
z \\
1
\end{array}\right) \frac{1}{2 \pi i} \int_{\mathbb{C}} d u \wedge d \bar{u} u^{2(h+1 / 2)-1} \bar{u}^{2 \bar{h}-1} A .
$$

We conclude that, when transformed to $\mathcal{C} \mathcal{S}^{2}, \lambda_{\alpha}$ is represented by the operator ${ }^{3} \lambda_{\alpha}=\left(\begin{array}{c}z \\ 1\end{array}\right) e^{\frac{1}{2} \partial_{h}}$, where the exponential operator shifts $h \rightarrow h+\frac{1}{2}$. Thus we find

$$
\begin{aligned}
\lambda_{\alpha} & =\left(\begin{array}{c}
z \\
1
\end{array}\right) e^{\frac{1}{2} \partial_{h}}, \quad \tilde{\lambda}_{\dot{\alpha}}=\left(\begin{array}{c}
\bar{z} \\
1
\end{array}\right) e^{\frac{1}{2} \partial_{\bar{h}}} \\
\partial_{\alpha} & =-\left(\begin{array}{c}
2 h-1+z \partial \\
\partial
\end{array}\right) e^{-\frac{1}{2} \partial_{h}}, \quad \tilde{\partial}_{\dot{\alpha}}=-\left(\begin{array}{c}
2 \bar{h}-1+\bar{z} \bar{\partial} \\
\bar{\partial}
\end{array}\right) e^{-\frac{1}{2} \partial_{\bar{h}}} .
\end{aligned}
$$

We can then immediately write down the generators of the conformal algebra from their momentum space counterparts using the replacements (4.3).

These are:

$$
\begin{gathered}
p_{\alpha \dot{\alpha}}=\lambda_{\alpha} \tilde{\lambda}_{\dot{\alpha}}=\left(\begin{array}{cc}
z \bar{z} & z \\
\bar{z} & 1
\end{array}\right)_{\alpha \dot{\alpha}} e^{\frac{1}{2}\left(\partial_{h}+\partial_{\bar{h}}\right)}, \\
m_{\alpha \beta}=\lambda_{(\alpha} \partial_{\beta)}=\left(\begin{array}{cc}
-2 z h-z^{2} \partial & -h-z \partial \\
-h-z \partial & -\partial
\end{array}\right)_{\alpha \beta}, \\
\bar{m}_{\dot{\alpha} \dot{\beta}}=\tilde{\lambda}_{(\dot{\alpha}} \tilde{\partial}_{\dot{\beta})}=\left(\begin{array}{cc}
-2 \bar{z} \bar{h}-\bar{z}^{2} \bar{\partial} & -\bar{h}-\bar{z} \bar{\partial} \\
-\bar{h}-\bar{z} \bar{\partial} & -\bar{\partial}
\end{array}\right)_{\dot{\alpha} \dot{\beta}} \\
k_{\alpha \dot{\alpha}}=\partial_{\alpha} \tilde{\partial}_{\dot{\alpha}}=\left(\begin{array}{cc}
(2 h-1+z \partial)(2 \bar{h}-1+\bar{z} \bar{\partial}) & (2 h-1+z \partial) \bar{\partial} \\
(2 \bar{h}-1+\bar{z} \bar{\partial}) \partial & \partial \bar{\partial}
\end{array}\right)_{\alpha \dot{\alpha}} e^{-\frac{1}{2}\left(\partial_{h}+\partial_{\bar{h}}\right)}, \\
d=\frac{1}{2} \lambda^{\alpha} \partial_{\alpha}+\frac{1}{2} \tilde{\lambda}^{\dot{\alpha}} \tilde{\partial}_{\dot{\alpha}}+1=-(h+\bar{h}-1) .
\end{gathered}
$$

These match the generators given in [39]. Note that they automatically satisfy the conformal algebra. ${ }^{4}$ This follows from the fact that the celestial space operators representing the spinors and the spinor derivatives have the same basic commutation relations as the original momentum space spinors and their derivatives.

\footnotetext{
${ }^{3}$ We use the same symbols for the operator representations of objects in both momentum space and celestial space; the difference will be clear from the context.

${ }^{4}$ The superconformal algebra of $\mathcal{N}=4 \mathrm{SYM}$ can be found in e.g., [1,41].
} 
The last property we wish to check is that the celestial conformal generators annihilate conformally invariant amplitudes, e.g., tree amplitudes in pure Yang-Mills theory. Once again this is immediate: all the celestial generators above have the property that we can push them back through the Mellin transform to obtain the original momentum space versions. In summary, the celestial generators satisfy all of the properties one would expect: they close the algebra and annihilate amplitudes.

We comment that deriving these generators using nonchiral coordinates is not as straightforward as above, as it involves relating four variables, $\left\{\lambda_{\alpha}, \tilde{\lambda}_{\dot{\alpha}}\right\}$, to three, $\{z, \bar{z}, \omega\}$. To fix this in nonchiral coordinates we must require

$\lambda_{\alpha}=\sqrt{\omega}\left(\begin{array}{c}z \\ 1\end{array}\right), \quad \tilde{\lambda}_{\dot{\alpha}}=\sqrt{\omega}\left(\begin{array}{c}\bar{z} \\ 1\end{array}\right) \Rightarrow \lambda_{2}=\tilde{\lambda}_{\dot{2}}$

Due to this constraint, the spinors $\lambda_{\alpha}$ and $\tilde{\lambda}_{\dot{\alpha}}$, as well as their derivatives $\partial_{\alpha}, \tilde{\partial}_{\dot{\alpha}}$, can no longer be treated as independent, which considerably complicates the analysis.

\section{B. Weights of celestial operators}

In using chiral coordinates we no longer have explicit weight factors appearing when we perform a Lorentz transformation in momentum space [see (2.16), as opposed to its nonchiral counterpart (2.10)]. Celestial amplitudes and the spinors they are built from will of course still have weights under the two-dimensional conformal group on the celestial sphere. By relating the chiral Mellin transform (which treats $h$ and $\bar{h}$ as free parameters) to the nonchiral Mellin transform, the weights $h, \bar{h}$ of a celestial amplitude were found in Sec. III A to be constrained to be $(h, \bar{h})=\left(\frac{\Delta+J}{2}, \frac{\Delta-J}{2}\right)$. We now show how this mechanism also assigns weights to the celestial conformal generators according to their helicity.

Consider a celestial spinor acting on a state $|z, \bar{z}, h, \bar{h} ; J\rangle$,

$$
\begin{aligned}
\lambda_{\alpha}|z, \bar{z}, h, \bar{h} ; J\rangle= & \frac{1}{2 \pi i} \int_{\mathbb{C}} d u \wedge d \bar{u} u^{2 h-1} \bar{u}^{2 \bar{h}-1} u\left(\begin{array}{l}
z \\
1
\end{array}\right)_{\alpha} \\
& \times|z, \bar{z}, u, \bar{u} ; J\rangle .
\end{aligned}
$$

Under a Lorentz transformation, spinors and states transform as in (2.16) and (3.12), respectively, hence under a Lorentz transformation we have

$$
\begin{aligned}
\lambda_{\alpha}|z, \bar{z}, h, \bar{h} ; J\rangle & \rightarrow \frac{1}{2 \pi i} \int_{\mathbb{C}} d u \wedge d \bar{u} u^{2 h-1} \bar{u}^{2 \bar{h}-1} u^{\prime}\left(\begin{array}{c}
z^{\prime} \\
1
\end{array}\right)_{\alpha}\left|z^{\prime}, \bar{z}^{\prime}, u^{\prime}, \bar{u}^{\prime} ; J\right\rangle \\
& =(c z+d)^{-2 h}(\bar{c} \bar{z}+\bar{d})^{-2 \bar{h}} \frac{1}{2 \pi i} \int_{\mathbb{C}} d u^{\prime} \wedge d \bar{u}^{\prime} u^{\prime 2 h} \bar{u}^{\prime 2 \bar{h}-1}\left(\begin{array}{c}
z^{\prime} \\
1
\end{array}\right)_{\alpha}\left|z^{\prime}, \bar{z}^{\prime}, u^{\prime}, \bar{u}^{\prime} ; J\right\rangle \\
& =(c z+d)^{-2 h}(\bar{c} \bar{z}+\bar{d})^{-2 \bar{h}} \lambda_{\alpha}^{\prime}\left|z^{\prime}, \bar{z}^{\prime}, h, \bar{h} ; J\right\rangle,
\end{aligned}
$$

where in the last line $\lambda_{\alpha}^{\prime}=\left(\begin{array}{c}z^{\prime} \\ 1\end{array}\right)_{\alpha} e^{\frac{1}{2} \partial_{h}}$. From (4.11) we see that the celestial spinor operator acting on a state, $\lambda_{\alpha}|z, \bar{z}, u, \bar{u} ; J\rangle$, has seemingly the same weight factors as $|z, \bar{z}, u, \bar{u} ; J\rangle$ and this is also the case for all of the chiral operators in (4.3). This is an incorrect conclusion. Indeed, this argument misses the fact that the parameters $h, \bar{h}$ must acquire values that satisfy the spin constraint arising from the Kronecker delta. As such, to ascertain the weights of $\lambda_{\alpha}|z, \bar{z}, u, \bar{u} ; J\rangle$ we must also consider the action of the operator $\lambda_{\alpha}$ on the spin constraint. We now consider the action of a spinor on a state again, making the connection to the nonchiral Mellin transform explicit,

$$
\begin{aligned}
\lambda_{\alpha}|z, \bar{z}, h, \bar{h} ; J\rangle & =\left(\begin{array}{c}
z \\
1
\end{array}\right)_{\alpha} e^{\frac{1}{2} \partial_{h}} \delta_{h-\bar{h}-J, 0}|z, \bar{z}, \Delta ; J\rangle \\
& =\left(\begin{array}{c}
z \\
1
\end{array}\right)_{\alpha} \delta_{h-\bar{h}-\left(J-\frac{1}{2}\right), 0} \int_{0}^{\infty} d \omega \omega^{h+\bar{h}+\frac{1}{2}-1}|z, \bar{z}, \omega ; J\rangle \\
& =\left(\begin{array}{c}
z \\
1
\end{array}\right)_{\alpha} \delta_{h-\bar{h}-\left(J-\frac{1}{2}\right), 0}\left|z, \bar{z}, \Delta+\frac{1}{2} ; J\right\rangle .
\end{aligned}
$$

From (4.12) we can read off

$$
h-\bar{h}=J-\frac{1}{2}, \quad h+\bar{h}+\frac{1}{2}=\Delta+\frac{1}{2},
$$

therefore 


$$
h=\frac{\Delta+J}{2}-\frac{1}{4}, \quad \bar{h}=\frac{\Delta-J}{2}+\frac{1}{4} .
$$

These values can now be plugged in (4.11) to find the weights of the operator $\lambda_{\alpha}$ in celestial space. From this we see that $\lambda_{\alpha}$ has shifted the weights by $\left(-\frac{1}{4}, \frac{1}{4}\right)$, as expected from an object with helicity $-\frac{1}{2}$. This carries over to the other celestial generators, and we provide further examples in the Appendix along with a complete table of weights, Table I. Note that generators such as $p_{\alpha \dot{\alpha}}$ and $k_{\alpha \dot{\alpha}}$ are weightless since they do not feature operators that act on the spin constraint $h-\bar{h}=J$.

\section{FROM ON-SHELL SUPERSPACE TO CELESTIAL SUPERAMPLITUDES}

\section{A. Lightning review of $\mathcal{N}=4$ superamplitudes}

We begin with a short review of the amplitudes in $\mathcal{N}=4$ SYM. In this theory one can package all amplitudes with fixed total helicity and number of particles $n$ into a superamplitude. This depends on some auxiliary Graßmann variables $\eta_{i}^{A}$, one for each particle (labeled by $i$ ), with $A=1, \ldots, 4$ being an index of the fundamental representation of the SU(4) $4 R$-symmetry group. By expanding a superamplitude in these fermionic variables, one can obtain the various component amplitudes. The precise correspondence can be derived from the Nair super-creation operator (often called the Nair superwavefunction) [42] which incorporates the creation operators of the physical states,

$$
\begin{aligned}
\Phi(\lambda, \tilde{\lambda}, \eta)= & G_{+}+\eta^{A} \Gamma_{A}+\frac{1}{2 !} \eta^{A} \eta^{B} S_{A B}+\frac{1}{3 !} \varepsilon_{A B C D} \eta^{B} \eta^{C} \eta^{D} \bar{\Gamma}^{A} \\
& +\frac{1}{4 !} \varepsilon_{A B C D} \eta^{A} \eta^{B} \eta^{C} \eta^{D} G_{-}
\end{aligned}
$$

Then, in order to select a state with helicity $J_{i}$, one needs to pick the term with $2-2 J_{i}$ powers of $\eta_{i}$ in the Taylor expansion of the superamplitude. Note that $\Phi(p, \eta)$ has uniform helicity +1 since we assign the $\eta^{A}$ variables helicity $+1 / 2$.

$\mathcal{N}=4 \mathrm{SYM}$ is a superconformal theory, and we now briefly review the generators of this symmetry. Firstly, there are the supersymmetry generators $q_{\alpha}^{A}, \bar{q}_{\dot{\alpha} A}$, which satisfy

$$
\left\{q_{\alpha}^{A}, \bar{q}_{\dot{\alpha} B}\right\}=\delta_{B}^{A} \lambda_{\alpha} \tilde{\lambda}_{\dot{\alpha}} .
$$

Importantly, on-shell superspace allows us to realize these generators as

$$
q_{\alpha}^{A}=\lambda_{\alpha} \eta^{A}, \quad \bar{q}_{\dot{\alpha} A}=\tilde{\lambda}_{\dot{\alpha}} \partial_{A} .
$$

In addition we have the generators of dilations and special conformal transformations [43]

$$
d=\frac{1}{2} \lambda^{\alpha} \partial_{\alpha}+\frac{1}{2} \tilde{\lambda}^{\dot{\alpha}} \partial_{\alpha}+1, \quad k_{\alpha \dot{\alpha}}=\partial_{\alpha} \partial_{\dot{\alpha}},
$$

together with two conformal supersymmetry generators

$$
s_{\alpha A}=\partial_{\alpha} \partial_{A}, \quad \bar{s}_{\dot{\alpha}}^{A}=\partial_{\dot{\alpha}} \eta^{A} .
$$

Since the helicity of each supermultiplet created by $\Phi(\lambda, \tilde{\lambda}, \eta)$ is equal to one, the operator

$$
\mathcal{J}=\frac{1}{2}\left(-\lambda^{\alpha} \partial_{\alpha}+\tilde{\lambda}^{\dot{\alpha}} \partial_{\dot{\alpha}}+\eta^{A} \partial_{A}\right),
$$

is such that for each particle $i$ one has [43]

$$
\mathcal{J}_{i} \mathcal{A}_{n}\left(\left\{\lambda_{j}, \tilde{\lambda}_{j}, \eta_{j}\right\}\right)=\mathcal{A}_{n}\left(\left\{\lambda_{j}, \tilde{\lambda}_{j}, \eta_{j}\right\}\right) .
$$

The algebra also has a central charge operator $c=1-\mathcal{J}$ which for each particle annihilates the superamplitude:

$$
c_{i} \mathcal{A}_{n}\left(\left\{\lambda_{j}, \tilde{\lambda}_{j}, \eta_{j}\right\}\right)=0 .
$$

Finally the SU(4)4 generators are

$$
r_{B}^{A}=\eta^{A} \partial_{B}-\frac{1}{4} \delta_{B}^{A} \eta^{C} \partial_{C} .
$$

Our next task is to find expressions for these generators on the celestial sphere.

\section{B. Celestial on-shell superspace}

To consider celestial superamplitudes we must first extend the celestial sphere to include a Graßmann variable and hence define a $\mathcal{C S}^{2}$ superspace. This was done for the $\mathcal{N}=1$ case in [27], we now do this for $\mathcal{N}=4$ SYM using chiral coordinates in the on-shell superspace formalism reviewed in Sec. VA.

To begin with, we consider a Mellin transform of the superwavefunction (5.1), as we did in Sec. III B for singleparticle states. However, before we do this we must consider the transformation properties of $\eta^{A}$, which is invariant under a Lorentz transformation, and transforms under a little group rotation of the corresponding particle since it carries helicity $+1 / 2$. Thus, in a similar manner to the positive-helicity spinor $\tilde{\lambda}_{\dot{\alpha}}$, we replace $\eta^{A}$ in terms of a new Graßmann variable $\tau^{A}$ which is invariant under the little group together with a phase that carries the little group transformation:

$$
\eta^{A} \rightarrow e^{-i \theta} \tau^{A}=\sqrt{\frac{\bar{u}}{u}} \tau^{A} .
$$

Using the Lorentz invariance of $\eta^{A}$, the definition (5.10) and (2.17), we see that $\tau^{A}$ must change with the following Möbius transformation under a Lorentz transformation:

$$
\tau^{\prime A}=\left(\frac{c z+d}{\bar{c} \bar{z}+\bar{d}}\right)^{\frac{1}{2}} \tau^{A},
$$


while it is by construction invariant under the little group. Note that (5.11) parallels (2.10).

From here we define the following conformal primary celestial superwavefunction:

$$
\tilde{\Phi}(z, \bar{z}, h, \bar{h}, \tau):=\frac{1}{2 \pi i} \int_{\mathbb{C}} d u \wedge d \bar{u} u^{2 h-1} \bar{u}^{2 \bar{h}-1}\left(|z, \bar{z}, u, \bar{u},+1\rangle+\eta^{A}|z, \bar{z}, u, \bar{u},+1 / 2\rangle_{A}+\cdots\right),
$$

where on the right-hand side of this equation $\eta$ should be regarded as the function of $\tau$ obtained by performing the replacement in (5.10). The reason behind this definition becomes clear once we relate this to the nonchiral Mellin transform. Indeed, doing so we see that the superwavefunction (and hence the superamplitudes) has a uniform helicity weight +1 :

$$
\tilde{\Phi}(z, \bar{z}, h, \bar{h}, \tau)=\delta_{h-\bar{h}-1,0} \int_{0}^{\infty} d \omega \omega^{h+\bar{h}-1}\left(|z, \bar{z}, \omega,+1\rangle+\tau^{A}|z, \bar{z}, \omega,+1 / 2\rangle_{A}+\cdots\right),
$$

where we have used (3.3). Note that had we not performed the replacement (5.10) then each term in the superwavefunction would have different weights, and we could not have interpreted it as a conformal primary.

As with the spinors and their derivatives, we can write $\eta_{A}$ and $\partial_{A}$ as operators on the celestial sphere by pulling them through the Mellin transform

$$
\eta^{A}=\tau^{A} e^{\left(-\partial_{h}+\partial_{\bar{h}}\right) / 4}, \quad \partial_{A}=\check{\partial}_{A} e^{\left(\partial_{h}-\partial_{\bar{h}}\right) / 4},
$$

where we define $\breve{\partial}_{A}=\frac{\partial}{\partial \tau^{A}}$. Using these operators we can extract the conformal primaries for all particles from the celestial superwavefunction by simply taking fermionic derivatives. For example, for the gluino we have

$$
\begin{aligned}
|z, \bar{z}, h, \bar{h},+1 / 2\rangle_{A} & =\left.\partial_{A} \tilde{\Phi}(z, \bar{z}, h, \bar{h}, \tau)\right|_{\tau=0}=\left.\check{\partial}_{A} e^{\left(\partial_{h}-\partial_{\bar{h}}\right) / 4} \tilde{\Phi}(z, \bar{z}, h, \bar{h}, \tau)\right|_{\tau=0} \\
& =\delta_{h-\bar{h}-\frac{1}{2}, 0} \int_{0}^{\infty} d \omega \omega^{h+\bar{h}-1}|z, \bar{z}, \omega,+1 / 2\rangle_{A} .
\end{aligned}
$$

\section{Celestial superamplitudes}

The chiral Mellin transform of a superamplitude proceeds as a natural extension of the nonsupersymmetric case. A celestial superamplitude is given by

$$
\tilde{\mathcal{A}}_{n}\left(\left\{z_{i}, \bar{z}_{i}, h_{i}, \bar{h}_{i}, \tau_{i}\right\}\right):=\frac{1}{(2 \pi i)^{n}} \prod_{i=1}^{n} \int_{\mathbb{C}} d u_{i} \wedge d \bar{u}_{i} u_{i}^{2 h_{i}-1} \bar{u}_{i}^{2 \bar{h}_{i}-1} \mathcal{A}_{n}\left(\left\{u_{i}, \bar{u}_{i}, z_{i}, \bar{z}_{i}, \sqrt{\frac{\bar{u}_{i}}{u_{i}}} \tau_{i}\right\}\right) .
$$

We can again relate this to the nonchiral Mellin transform in the $\omega$ coordinate similarly to what was done in Sec. III C. The result is

$$
\tilde{\mathcal{A}}_{n}\left(\left\{z_{i}, \bar{z}_{i}, h_{i}, \bar{h}_{i}, \tau_{i}\right\}\right)=\prod_{i=1}^{n} \delta_{h_{i}-\bar{h}_{i}-1,0} \int_{0}^{\infty} d \omega_{i} \omega_{i}^{h_{i}+\bar{h}_{i}-1} \mathcal{A}_{n}\left(\left\{\omega_{i}, z_{i}, \bar{z}_{i}, \tau_{i}\right\}\right)
$$

where we have used (5.7). Note the appearance of Kronecker deltas enforcing the spin constraint $h_{i}-\bar{h}_{i}=1$ for every particle $i$. (5.17) is the supersymmetric generalization of (3.14). On the support of the Kronecker delta, the celestial superamplitude transforms as a two-dimensional conformal correlator with weights now given by $\left(h_{i}, \bar{h}_{i}\right)=\left(\frac{\Delta_{i}+1}{2}, \frac{\Delta_{i}-1}{2}\right)$. Under Lorentz transformations we have

$$
\tilde{\mathcal{A}}_{n}\left(\left\{z_{i}, \bar{z}_{i}, h_{i}, \bar{h}_{i}, \tau_{i}\right\}\right)=\prod_{i=1}^{n}\left(c z_{i}+d\right)^{-\Delta_{i}-1}\left(\bar{c} \bar{z}_{i}+\bar{d}\right)^{-\Delta_{i}+1} \tilde{\mathcal{A}}_{n}\left(\left\{\frac{a z_{i}+b}{c z_{i}+d}, \frac{\bar{a} \bar{z}+\bar{b}}{\bar{c} \bar{z}+\bar{d}}, h_{i}, \bar{h}_{i},\left(\frac{\bar{c} \bar{z}+\bar{d}}{c z+d}\right)^{-\frac{1}{2}} \tau_{i}\right\}\right) .
$$

We can examine the infinitesimal version of (5.18) to obtain the generators of the Lorentz symmetry on celestial superamplitudes. In order to do so we first note that a Möbius transformation (2.8) is now accompanied by the transformation $\tau \rightarrow \tau^{\prime A}$ defined in (5.11). The infinitesimal transformation has then the form

$$
z^{\prime}=z+\epsilon(z), \quad \bar{z}^{\prime}=\bar{z}+\bar{\epsilon}(\bar{z}), \quad \tau^{\prime}=\tau+\kappa(z, \bar{z}) \tau,
$$

with $\kappa=\frac{1}{4} \bar{\partial} \bar{\epsilon}-\frac{1}{4} \partial \epsilon$. The corresponding generator acting on each particle is then (omitting the particle label) 


$$
\begin{aligned}
& h \partial \epsilon+\epsilon \partial+\bar{h} \bar{\partial} \bar{\epsilon}+\bar{\epsilon} \bar{\partial}+\kappa \tau^{A} \check{\partial}_{A} \\
& =\left(h-\frac{1}{4} \tau^{A} \check{\partial}_{A}\right) \partial \epsilon+\epsilon \partial+\left(\bar{h}+\frac{1}{4} \tau^{A} \check{\partial}_{A}\right) \bar{\partial} \bar{\epsilon}+\bar{\epsilon} \bar{\partial} .
\end{aligned}
$$

Thus we obtain the celestial superspace generators from those defined earlier in celestial space by simply shifting $h$ and $\bar{h}$ as

$$
h \rightarrow h-\frac{1}{4} \tau^{A} \check{\partial}_{A}, \quad \bar{h} \rightarrow \bar{h}+\frac{1}{4} \tau^{A} \check{\partial}_{A} .
$$

As an example, we have the following Lorentz generators acting on superamplitudes:

$$
\begin{gathered}
m_{\alpha \beta}=\left(\begin{array}{cc}
-2 z\left(h-\frac{1}{4} \tau^{A} \check{\partial}_{A}\right)-z^{2} \partial & -\left(h-\frac{1}{4} \tau^{A} \check{\partial}_{A}\right)-z \partial \\
-\left(h-\frac{1}{4} \tau^{A} \check{\partial}_{A}\right)-z \partial & -\partial
\end{array}\right)_{\alpha \beta}, \\
\bar{m}_{\dot{\alpha} \dot{\beta}}=\left(\begin{array}{cc}
-2 \bar{z}\left(\bar{h}+\frac{1}{4} \tau^{A} \check{\partial}_{A}\right)-\bar{z}^{2} \bar{\partial} & -\left(\bar{h}+\frac{1}{4} \tau^{A} \check{\partial}_{A}\right)-\bar{z} \bar{\partial} \\
-\left(\bar{h}+\frac{1}{4} \tau^{A} \check{\partial}_{A}\right)-\bar{z} \bar{\partial} & -\bar{\partial}
\end{array}\right)_{\dot{\alpha} \dot{\beta}} .
\end{gathered}
$$

These generators feature the weights of the superamplitude $(h, \bar{h})=\left(\frac{\Delta+1}{2}, \frac{\Delta-1}{2}\right)$ but shifted in such a way as to subtract off the weights of $\tau^{A}$ (or equivalently $\eta^{A}$ ), which, since it carries helicity $+\frac{1}{2}$, are just $\left(\frac{1}{4},-\frac{1}{4}\right)$. In these generators, the weight-counting operator acts on each term in the superamplitude and measures the weights of only the component amplitude in that term. Indeed this must be the case since the Lorentz generators act on the spinors alone and annihilate the superamplitude precisely by annihilating each component amplitude.

Note that the combination $\tau^{A} \check{\partial}_{A}$ is little group invariant and therefore we can replace it with $\eta^{A} \partial_{A}$. We leave this expression as it is to highlight the role $\tau^{A}$ plays in the chiral coordinates and also in the celestial superconformal algebra in Sec. VI.

\section{Examples of celestial superamplitudes}

Here we derive the supersymmetric three-, four- and fivepoint tree-level MHV superamplitudes using the Mellin transform. ${ }^{5}$ We also make some remarks on the general case. We will work with the chiral Mellin transform using its relationship with the standard nonchiral transform (3.14). To begin with we write the three-point MHV tree-level superamplitude in terms of nonchiral celestial coordinates,

$$
\begin{aligned}
\mathcal{A}_{3}^{\mathrm{MHV}} & =\delta^{(4)}\left(p_{1}+p_{2}-p_{3}\right) \frac{\delta^{(8)}\left(q_{1 \alpha}^{A}+q_{2 \alpha}^{A}-q_{3 \alpha}^{A}\right)}{\langle 12\rangle\langle 23\rangle\langle 31\rangle} \\
& =\delta^{(4)}\left(p_{1}+p_{2}-p_{3}\right) \frac{\delta^{(8)}\left(\sqrt{\omega_{1}} \xi_{1} \tau_{1}^{A}+\sqrt{\omega_{2}} \xi_{2} \tau_{2}^{A}-\sqrt{\omega_{3}} \xi_{3} \tau_{3}^{A}\right)}{-\omega_{1} \omega_{2} \omega_{3} z_{12} z_{23} z_{31}},
\end{aligned}
$$

where we take particles 1 and 2 incoming and 3 outgoing. To evaluate the Mellin transform with respect to each $\omega_{i}$ we first rewrite the momentum-conserving delta function as follows:

$$
\begin{aligned}
\delta^{(4)}\left(p_{1}+p_{2}-p_{3}\right) & =\delta^{(4)}\left(\omega_{1} \xi_{1} \tilde{\xi}_{1}+\omega_{2} \xi_{2} \tilde{\xi}_{2}-\omega_{3} \xi_{3} \tilde{\xi}_{3}\right) \\
& =\frac{4}{\omega_{3}^{2} z_{23} z_{31}} \delta\left(\bar{z}_{31}\right) \delta\left(\bar{z}_{23}\right) \delta\left(\omega_{1}-\omega_{3} \frac{z_{23}}{z_{21}}\right) \delta\left(\omega_{2}-\omega_{3} \frac{z_{31}}{z_{21}}\right) .
\end{aligned}
$$

The first two delta functions here imply the constraint $\tilde{\lambda}_{1} \sim \tilde{\lambda}_{2} \sim \tilde{\lambda}_{3}$ as expected for three-point MHV amplitudes. ${ }^{6}$ The remaining two delta functions trivialize the $\omega_{1}$ and $\omega_{2}$ integrals to give

$$
\begin{aligned}
\tilde{\mathcal{A}}_{3}^{\mathrm{MHV}} & =\prod_{i=1}^{3} \delta_{h_{-} \bar{h}_{i}-1,0} \int_{0}^{\infty} d \omega_{i} \omega_{i}^{\Delta_{i}-1} \mathcal{A}_{3}^{\mathrm{MHV}} \\
& =-4 \prod_{i=1}^{3} \delta_{h_{-} \bar{h}_{i}-1,0} \int_{0}^{\infty} d \omega_{3} \omega_{3}^{\left(\sum_{j=1}^{3} \Delta_{j}\right)-4} \delta\left(\bar{z}_{31}\right) \delta\left(\bar{z}_{23}\right) z_{21}^{3-\Delta_{1}-\Delta_{2}} z_{23}^{\Delta_{1}-4} z_{31}^{\Delta_{2}-4} \delta^{(8)}\left(\sqrt{\frac{z_{23}}{z_{21}}} \xi_{1} \tau_{1}^{A}+\sqrt{\frac{z_{31}}{z_{21}}} \xi_{2} \tau_{2}^{A}-\xi_{3} \tau_{3}^{A}\right) .
\end{aligned}
$$

\footnotetext{
${ }^{5}$ This section on explicit MHV celestial superamplitudes partly overlaps with work carried out recently in [40] although the formulæ at four- and five-points, (5.33) and (5.36), are new.

${ }^{6}$ Technically this means we must work with complexified momenta with $z$ and $\bar{z}$ independent at three points.
} 
To ensure that the conformal primary wavefunctions are delta-function normalizable we must have $\Delta_{i}=1+i \beta_{i}[10]$, hence the amplitude becomes

$$
\begin{aligned}
\tilde{\mathcal{A}}_{3}^{\mathrm{MHV}}= & -(8 \pi) \prod_{i=1}^{3} \delta_{h_{i}-\bar{h}_{i}-1,0} \delta\left(\sum_{i=1}^{3} \beta_{i}\right) \delta\left(\bar{z}_{31}\right) \delta\left(\bar{z}_{23}\right) z_{21}^{\Delta_{3}-4} z_{23}^{\Delta_{1}-4} z_{31}^{\Delta_{2}-4} \\
& \times \delta^{(8)}\left(\sqrt{z_{23}} \xi_{1} \tau_{1}^{A}+\sqrt{z_{31}} \xi_{2} \tau_{2}^{A}-\sqrt{z_{21}} \xi_{3} \tau_{3}^{A}\right) \\
= & -(8 \pi) \prod_{i=1}^{3} \delta_{h_{i}-\bar{h}_{i}-1,0} \delta\left(\sum_{i=1}^{3} \beta_{i}\right) \delta\left(\bar{z}_{31}\right) \delta\left(\bar{z}_{23}\right) \prod_{i<j} z_{i j}^{3-2 h_{i}-2 h_{j}} \\
& \times \delta^{(8)}\left(\left(\frac{z_{23}}{z_{31} z_{12}}\right)^{1 / 4} \xi_{1} \tau_{1}^{A}+\left(\frac{z_{31}}{z_{12} z_{23}}\right)^{1 / 4} \xi_{2} \tau_{2}^{A}-\left(\frac{z_{12}}{z_{23} z_{31}}\right)^{1 / 4} \xi_{3} \tau_{3}^{A}\right),
\end{aligned}
$$

where in the last expression we have extracted the term carrying the conformal scaling properties for the variables $z$. To check this result we can extract the three-point MHV gluon amplitude from the superamplitude by acting with the appropriate Graßmann derivatives $\partial_{A}=e^{\left(\partial_{h}-\partial_{\bar{h}}\right) / 4} \check{\partial}_{A}$,

$$
\begin{aligned}
\tilde{A}_{3}^{\mathrm{MHV}}(-,-,+) & =\left(\partial_{1 A}\right)^{4}\left(\partial_{2 A}\right)^{4} \tilde{\mathcal{A}}^{\mathrm{MHV}}=\left(\check{\partial}_{1 A}\right)^{4}\left(\check{\partial}_{2 A}\right)^{4} e^{\left(\partial_{h_{1}}-\partial_{\bar{h}_{1}}\right)} e^{\left(\partial_{h_{2}}-\partial_{\bar{h}_{2}}\right)} \tilde{\mathcal{A}}^{\mathrm{MHV}} \\
& =-(8 \pi) \delta_{h_{1}-\bar{h}_{1}+1,0} \delta_{h_{2}-\bar{h}_{2}+1,0} \delta_{h_{3}-\bar{h}_{3}-1,0} \delta\left(\sum_{i=1}^{3} \beta_{i}\right) \delta\left(\bar{z}_{31}\right) \delta\left(\bar{z}_{23}\right) z_{21}^{\Delta_{3}} z_{23}^{\Delta_{1}-2} z_{31}^{\Delta_{2}-2}
\end{aligned}
$$

If we strip off the helicity constraint deltas from each leg this result agrees with [12] up to an overall normalization.

We can follow a similar calculation for the four-point MHV superamplitude. As before, we rewrite the momentumconserving delta function

$$
\begin{aligned}
\delta^{(4)}\left(p_{1}+p_{2}-p_{3}-p_{4}\right) & =\delta^{(4)}\left(\omega_{1} \xi_{1} \tilde{\xi}_{1}+\omega_{2} \xi_{2} \tilde{\xi}_{2}-\omega_{3} \xi_{3} \tilde{\xi}_{3}-\omega_{4} \xi_{4} \tilde{\xi}_{4}\right) \\
& =\frac{4 \delta(r-\bar{r})}{\omega_{1}\left|z_{13}\right|^{2}\left|z_{24}\right|^{2}} \delta\left(\omega_{4}+\omega_{1} \frac{z_{13} \bar{z}_{12}}{z_{34} \bar{z}_{24}}\right) \delta\left(\omega_{3}-\omega_{1} \frac{z_{14} \bar{z}_{12}}{z_{34} \bar{z}_{23}}\right) \delta\left(\omega_{2}+\omega_{1} \frac{z_{13} \bar{z}_{14}}{z_{23} \bar{z}_{24}}\right)
\end{aligned}
$$

where $r$ is the cross-ratio given by

$$
r=\frac{z_{12} z_{34}}{z_{13} z_{24}}
$$

The $\delta(r-\bar{r})$ term imposes the constraint from $2 \rightarrow 2$ scattering that all four particles lie in the same plane [32]. Again we have three delta functions which trivialize the integrals over $\omega_{2,3,4}$ and the remaining integral over $\omega_{1}$ gives us a $\delta(\beta)$ factor, where $\beta=\sum_{i=1}^{4} \beta_{i}$. Thus the four-point celestial MHV superamplitude is

$$
\begin{aligned}
\tilde{\mathcal{A}}_{4}^{\mathrm{MHV}}= & (8 \pi) \prod_{i=1}^{4} \delta_{h_{i}-\bar{h}_{i}-1,0} \delta(\beta)\left(\frac{z_{13}}{\bar{z}_{42}}\right)^{\Delta_{2}+\Delta_{4}-4}\left(\frac{\bar{z}_{12}}{z_{34}}\right)^{\Delta_{3}+\Delta_{4}-4}\left(\frac{\bar{z}_{14}}{z_{23}}\right)^{\Delta_{2}-2}\left(\frac{z_{14}}{\bar{z}_{23}}\right)^{\Delta_{3}-2} \\
& \times \frac{\delta(r-\bar{r})}{\left.z_{12} z_{23} z_{34} z_{41}\left|z_{13}\right| z_{24}\right|^{2}} \delta^{(8)}\left(\xi_{1} \tau_{1}^{A}+\sqrt{\frac{z_{13} \bar{z}_{14}}{z_{23} \bar{z}_{42}}} \xi_{2} \tau_{2}^{A}-\sqrt{\frac{z_{14} \bar{z}_{12}}{z_{34} \bar{z}_{23}}} \xi_{3} \tau_{3}^{A}-\sqrt{\frac{z_{13} \bar{z}_{12}}{z_{34} \bar{z}_{42}}} \xi_{4} \tau^{4}\right) .
\end{aligned}
$$

Defining the conformally covariant expression

$$
I_{4}(z, \bar{z})=\prod_{i<j} z_{i j}^{\frac{h}{3}-\left(h_{i}+h_{j}\right)} \bar{z}_{i j}^{\frac{\bar{h}}{-}-\left(\bar{h}_{i}+\bar{h}_{j}\right)},
$$

where $h=\sum_{i=1}^{4} h_{i}$ and $\bar{h}=\sum_{i=1}^{4} \bar{h}_{i}$, and

$$
x_{i}=\prod_{j<k}\left(z_{j k} \bar{z}_{j k}\right)^{\frac{1}{12}} \prod_{l \neq i}\left(z_{i l} \bar{z}_{i l}\right)^{-\frac{1}{4}}=I_{4}^{-1} e^{\frac{\partial_{h_{i}}+\partial_{\bar{h}_{i}}}{4}} I_{4},
$$


then the above amplitude may be written as (with $\epsilon_{1}=\epsilon_{2}=-\epsilon_{3}=-\epsilon_{4}=1$ )

$$
\tilde{\mathcal{A}}_{4}^{\mathrm{MHV}}=(8 \pi) \prod_{i=1}^{4} \delta_{h_{i}-\bar{h}_{i}, 1} \delta(\beta) \delta(r-\bar{r}) I_{4}(z, \bar{z}) r^{\frac{1}{3}}(1-r)^{\frac{1}{3}} \delta^{(8)}\left(\sum_{i=1}^{4} \epsilon_{i} x_{i} \xi_{i} \tau_{i}^{A}\right) .
$$

Now we can write

$$
\delta^{8}\left(\sum_{i=1}^{4} \epsilon_{i} x_{i} \xi_{i \alpha} \tau_{i}^{A}\right)=2 \prod_{A=1}^{4} \sum_{i<j} \epsilon_{i} \epsilon_{j} x_{i} x_{j} z_{i j} \tau_{i}^{A} \tau_{j}^{A},
$$

and conclude that this fermionic delta function is weightless under $\left(z_{i}, \tau_{i}^{A}\right) \rightarrow\left(z_{i}^{\prime}, \tau_{i}^{\prime A}\right)$; this is only made possible using a supercoordinate $\tau^{A}$ which transforms under Möbius maps. The result (5.33) for the superamplitude is consistent with the four-point gluon amplitude appearing in, for example, [12].

Expressions for the bosonic $n$-point amplitudes have been derived in [21,30]. At five points, we define the conformally covariant expression

$$
I_{5}(z, \bar{z})=\prod_{i<j} z_{i j}^{\frac{h}{6} \frac{-2}{3}\left(h_{i}+h_{j}\right)} \bar{z}_{i j}^{\frac{\bar{b}}{6}-\frac{2}{3}\left(\bar{h}_{i}+\bar{h}_{j}\right)}
$$

Then we find that the five-point MHV superamplitude can be written in the form

$$
\tilde{\mathcal{A}}_{5}^{\mathrm{MHV}} \sim \prod_{i=1}^{5} \delta_{h_{i}-\bar{h}_{i}, 1} \delta(\beta) I_{5}(z, \bar{z}) X\left(r,\left\{h_{i}, \bar{h}_{i}\right\}\right) \delta^{(8)}\left(\sum_{i=1}^{5} \epsilon_{i} y_{i} V_{i}(r) \xi_{i} \tau_{i}^{A}\right)
$$

where

$$
y_{i}=\prod_{j<k}\left(z_{j k} \bar{z}_{j k}\right)^{\frac{1}{24}} \prod_{l \neq i}\left(z_{i l} \bar{z}_{i l}\right)^{-\frac{1}{6}}=I_{5}^{-1} e^{\frac{\partial_{h_{i}}+\partial_{\bar{h}_{i}}}{4}} I_{5},
$$

and

$$
V_{i}(r)=X^{-1} e^{\frac{\partial_{h_{i}}+\partial_{\bar{h}_{i}}}{4} X}
$$

Here $X\left(r,\left\{h_{i}, \bar{h}_{i}\right\}\right)$ is a function only of the cross-ratios (of which there are two at five-point) and the weights $\left\{h_{i}, \bar{h}_{i}\right\}$ which appear as exponents. The amplitude (5.36) transforms covariantly under two-dimensional conformal transformations with the correct weights.

For $n>5$ points, the bosonic amplitudes are given in terms of $n-4$ integrals. In the formulation of [21], one can show that there are changes of integration variables such that the $z, \bar{z}$ dependence inside the integrals is only through cross-ratios. For the superamplitudes, this approach can also be followed, and the outcome is that the superamplitude is given by integrals over expressions which take a form analogous to the right-hand side of (5.36), where the function $X$ and the coefficients inside the supersymmetric delta function now depend on the integration variables and the cross-ratios. These expressions are rather complicated and it would be of interest to explore if there is a more concise formulation.

\section{CELESTIAL SUPERCONFORMAL ALGEBRA}

With our description of celestial on-shell superspace in chiral coordinates and a chiral Mellin transform taking us to Mellin space, we can derive the generators of superconformal symmetry. We will follow the same path as in $\mathrm{Sec}$. IV to write the generators on the celestial sphere in terms of their momentum space counterparts.

Our first step is to write the spinors $\lambda_{\alpha}, \tilde{\lambda}_{\dot{\alpha}}$ and the supercoordinate $\eta^{A}$ in chiral coordinates, and perform a change of variables from $\left\{\lambda_{\alpha}, \tilde{\lambda}_{\dot{\alpha}}, \eta^{A}\right\}$ to $\left\{z, \bar{z}, u, \bar{u}, \tau^{A}\right\}$. Since $\eta^{A}=(\bar{u} / u)^{1 / 2} \tau^{A}$, the reparametrization of superspace in chiral coordinates will also change the form of the spinor derivatives, for example

$$
\begin{aligned}
\frac{\partial}{\partial \lambda^{1}} & =\frac{\partial z}{\partial \lambda^{1}} \frac{\partial}{\partial z}+\frac{\partial \bar{z}}{\partial \lambda^{1}} \frac{\partial}{\partial \bar{z}}+\frac{\partial u}{\partial \lambda^{1}} \frac{\partial}{\partial u}+\frac{\partial \bar{u}}{\partial \lambda^{1}} \frac{\partial}{\partial \bar{u}}+\frac{\partial \tau^{A}}{\partial \lambda^{1}} \frac{\partial}{\partial \tau^{A}} \\
& =-\frac{z}{u} \frac{\partial}{\partial z}+\frac{\partial}{\partial u}+\frac{1}{2} \frac{1}{u} \tau^{A} \frac{\partial}{\partial \tau^{A}}
\end{aligned}
$$

We see that $\tau^{A}$ now appears explicitly in the spinor derivatives, however this is not surprising given the form of the Lorentz generators we found in (5.22). For the remaining objects we have 


$$
\begin{aligned}
\lambda_{\alpha}=u\left(\begin{array}{l}
z \\
1
\end{array}\right)_{\alpha}, & \partial_{\alpha}=\frac{1}{u}\left(\begin{array}{c}
u \partial_{u}+\frac{1}{2} \tau^{A} \check{\partial}_{A}-z \partial \\
-\partial
\end{array}\right)_{\alpha}, \\
\tilde{\lambda}_{\dot{\alpha}}=u\left(\begin{array}{c}
\bar{z} \\
1
\end{array}\right)_{\dot{\alpha}}, & \tilde{\partial}_{\dot{\alpha}}=\frac{1}{\bar{u}}\left(\begin{array}{c}
\bar{u} \partial_{\bar{u}}-\frac{1}{2} \tau^{A} \check{\partial}_{A}-\bar{z} \bar{\partial}, \\
-\bar{\partial}
\end{array}\right)_{\alpha}, \\
\eta^{A}=\left(\frac{\bar{u}}{u}\right)^{1 / 2} \tau^{A}, & \partial_{A}=\left(\frac{\bar{u}}{u}\right)^{-1 / 2} \check{\partial}_{A} .
\end{aligned}
$$

As in Sec. IV, these are uniquely mapped to operators in Mellin space under the chiral Mellin transform. We obtain ${ }^{7}$

$$
\begin{aligned}
& \lambda_{\alpha}=\left(\begin{array}{c}
z \\
1
\end{array}\right)_{\alpha} e^{\frac{\partial_{h}}{2}}, \quad \partial_{\alpha}=-\left(\begin{array}{c}
2\left(h-\frac{1}{4} \tau^{A} \check{\partial}_{A}\right)-1+z \partial \\
\partial
\end{array}\right)_{\alpha} e^{-\frac{\partial_{h}}{2}}, \\
& \tilde{\lambda}_{\dot{\alpha}}=\left(\begin{array}{c}
\bar{z} \\
1
\end{array}\right)_{\dot{\alpha}} e^{\frac{\partial_{\bar{h}}}{2}}, \quad \tilde{\partial}_{\dot{\alpha}}=-\left(\begin{array}{c}
2\left(\bar{h}+\frac{1}{4} \tau^{A} \check{\partial}_{A}\right)-1+\bar{z} \bar{\partial}, \\
\bar{\partial}
\end{array}\right)_{\alpha} e^{-\frac{\partial_{\bar{h}}}{2}}, \\
& \eta^{A}=\tau^{A} e^{-\frac{\partial_{h}}{4}+\frac{\partial_{\bar{h}}}{4}}, \quad \partial_{A}=\check{\partial}_{A} e^{\frac{\partial_{h}}{4}-\frac{\partial_{\bar{h}}}{4}} .
\end{aligned}
$$

Note that $h$ and $\bar{h}$ are now shifted in the same way as in (5.21).

From here we can write down the superconformal generators on the celestial sphere, using their representation in on-shell superspace (as in e.g., $[1,41]$ ) and the dictionary provided by (6.3).

First we have translations, Lorentz, conformal and dilation generators:

$$
p_{\alpha \dot{\alpha}}=\lambda_{\alpha} \tilde{\lambda}_{\dot{\alpha}}=\left(\begin{array}{cc}
z \bar{z} & z \\
\bar{z} & 1
\end{array}\right)_{\alpha \dot{\alpha}} e^{\frac{\partial_{h}}{2}+\frac{\partial_{\bar{h}}}{2}}
$$

$$
\begin{aligned}
m_{\alpha \beta} & =\lambda_{(\alpha} \partial_{\beta)} \\
& =\left(\begin{array}{cc}
-2 z\left(h-\frac{1}{4} \tau^{A} \check{\partial}_{A}\right)-z^{2} \partial & -\left(h-\frac{1}{4} \tau^{A} \check{\partial}_{A}\right)-z \partial \\
-\left(h-\frac{1}{4} \tau^{A} \check{\partial}_{A}\right)-z \partial & -\partial
\end{array}\right)_{\alpha \beta}, \\
\bar{m}_{\dot{\alpha} \dot{\beta}} & =\tilde{\lambda}_{(\dot{\alpha}} \tilde{\partial}_{\beta \dot{\beta}} \\
& =\left(\begin{array}{cc}
-2 \bar{z}\left(\bar{h}+\frac{1}{4} \tau^{A} \check{\partial}_{A}\right)-\bar{z}^{2} \bar{\partial} & -\left(\bar{h}+\frac{1}{4} \tau^{A} \check{\partial}_{A}\right)-\bar{z} \bar{\partial} \\
-\left(\bar{h}+\frac{1}{4} \tau^{A} \check{\partial}_{A}\right)-\bar{z} \bar{\partial} & -\bar{\partial}
\end{array}\right)_{\dot{\alpha} \dot{\beta}},
\end{aligned}
$$

The $\mathcal{N}=4$ supersymmetry generators are given by:

$$
q_{\alpha}^{A}=\lambda_{\alpha} \eta^{A}=\left(\begin{array}{c}
z \\
1
\end{array}\right)_{\alpha} \tau^{A} e^{\frac{\partial_{h}}{4}+\frac{\partial_{\bar{h}}}{4}}, \quad \bar{q}_{\dot{\alpha} A}=\tilde{\lambda}_{\dot{\alpha}} \partial_{A}=\left(\begin{array}{c}
\bar{z} \\
1
\end{array}\right)_{\dot{\alpha}} \check{\partial}_{A} e^{\frac{\partial_{h}}{4}+\frac{\partial_{\bar{h}}}{4}} .
$$

This matches the structure of the $\mathcal{N}=1$ supersymmetry generators found in [27]. The remaining superconformal generators on the celestial sphere are

$$
\begin{gathered}
s_{\alpha A}=\partial_{\alpha} \partial_{A}=-\left(\begin{array}{c}
2\left(h-\frac{1}{4} \tau^{A} \check{\partial}_{A}\right)-1+z \partial \\
\partial
\end{array}\right)_{\alpha} \check{\partial}_{A} e^{-\frac{\partial_{h}}{4}-\frac{\partial_{\bar{h}}}{4}}, \\
\bar{s}_{\dot{\alpha}}^{A}=\tilde{\partial}_{\dot{\alpha}} \eta^{A}=-\left(\begin{array}{c}
2\left(\bar{h}+\frac{1}{4} \tau^{A} \check{\partial}_{A}\right)-1+\bar{z} \bar{\partial} \\
\bar{\partial}
\end{array}\right)_{\dot{\alpha}} \tau^{A} e^{-\frac{\partial_{h}}{4}-\frac{\partial_{\bar{h}}}{4}},
\end{gathered}
$$

\footnotetext{
${ }^{7}$ Again we use the same expression for a momentum space operator and its Mellin space counterpart.
}

$$
\begin{gathered}
r_{B}^{A}=\tau^{A} \check{\partial}_{B}-\frac{1}{4} \delta_{B}^{A} \tau^{C} \check{\partial}_{C}, \\
c:=1-\mathcal{J}=1-\left(h-\frac{1}{4} \tau^{A} \check{\partial}_{A}-\left(\bar{h}+\frac{1}{4} \tau^{A} \check{\partial}_{A}\right)+\frac{1}{2} \tau^{A} \check{\partial}_{A}\right) \\
=1-(h-\bar{h}) .
\end{gathered}
$$

One can deduce that these generators satisfy the full superconformal algebra by checking that the commutation relations of the celestial superspace operator representation of the variables $\left\{\lambda_{\alpha}, \tilde{\lambda}_{\dot{\alpha}}, \eta^{A}\right\}$ and the derivatives with respect to them reproduce those of the superspace variables and derivatives. These celestial superconformal generators are guaranteed to annihilate superamplitudes from their derivation, as was the case for the conformal generators in Sec. IV. 


\section{CONCLUSIONS}

In this paper we considered a celestial space parametrization of null momenta in which the little group is not fixed. This leads to a natural definition of a "chiral" Mellin transform which can be reduced to the usual "nonchiral" transform, but which also includes a spin constraint $h-\bar{h}=J$. We discussed how states and amplitudes are represented in these chiral celestial coordinates and how this approach simplifies the derivation of the conformal symmetry operators. We extended this discussion to $\mathcal{N}=4$ SYM, giving a definition of $\mathcal{N}=4$ chiral superspace, the states and superamplitudes, and presented some explicit examples. We further showed how the full superconformal algebra generators follow directly from their spacetime representations.

This work provides a potentially simplified approach to the study of (chiral) celestial amplitudes and their symmetries. It can be directly applied to the derivation of particular amplitudes and the formulation of recursion relations. Several avenues for further investigation are possible. Among these, one pressing question is to clarify the nature of a potential celestial two-dimensional superconformal field theory whose correlation functions are our superamplitudes of $\mathcal{N}=4 \mathrm{SYM}$. Extensions to supergravity, in particular $\mathcal{N}=8$ supergravity, should also be immediate, as should the study of amplitudes in theories with less than maximal supersymmetry, for which the appropriate on-shell superspace is known. It would also be very interesting to intensify the study of celestial loop amplitudes, and to find a possible celestial representation of hidden symmetries of the $\mathcal{N}=4$ SYM $S$-matrix such as the dual superconformal and Yangian symmetries. We will return to these questions in the near future.

\section{ACKNOWLEDGMENTS}

We would like to thank Rashid Alawadhi, Stefano De Angelis, Jung-Wook Kim, Adrian Keyo Shan Padellaro and Congkao Wen for interesting discussions. We also thank Hongliang Jiang for sharing his draft [44] with us, and Congkao Wen for informing each of us about this parallel work. This work was supported by the Science and Technology Facilities Council (STFC) Consolidated Grants No. ST/P000754/1 "String theory, gauge theory \& duality" and No. ST/T000686/1 "Amplitudes, strings \& duality" and by the European Union's Horizon 2020 research and innovation program under the Marie Skłodowska-Curie Grant agreement No. 764850 "SAGEX". The work of G. R. B. and J. G. is supported by an STFC quota studentship (Project Reference 2424584).

Note added.-After this work was completed we became aware that Hongliang Jiang, also at Queen Mary University of London, had been working independently, and unknowingly to us due to Covid restrictions, on very similar topics [44]. Happily the results presented in our two papers are in agreement although obtained in different ways.

\section{APPENDIX: CONFORMAL WEIGHTS}

In this Appendix we state the weights for all objects appearing in $\mathcal{N}=4 \mathrm{SYM}$, Table I, and provide some example derivations. The weights of an object are defined under the two-dimensional conformal group only once it has been mapped to Mellin space by a chiral Mellin transform, and are derived according to the method laid out in Sec. IV B.

To summarize, the weights of generators are always dictated by their helicity and carry zero conformal dimension-whereas splitting generators into their constituent differential operators produces objects with conformal dimension also. The weights of a celestial amplitude are given by the parameters in the chiral Mellin transform which have values $(h, \bar{h})=\left(\frac{\Delta+J}{2}, \frac{\Delta-J}{2}\right)$. Intuitively, the spin $J$ is set by the amplitude itself while the conformal dimension $\Delta:=h+\bar{h}$ is a free parameter set by the powers of $\omega$ (equivalently $u, \bar{u}$ ) in the Mellin transform. The weights of all operators are defined according to how they act on celestial amplitudes.

As examples we will now derive the weights of the Graßmann variable $\eta^{A}$, the spinor derivative $\partial_{\alpha}$ and the exponential operator $e^{\frac{\partial_{h}}{2}}$.

\section{Graßmann variable: $\eta^{A}$}

Following Sec. IV B we can compute the weights of the celestial operator $\eta^{A}=\tau^{A} e^{-\frac{\partial_{h}}{4}+\frac{\partial_{\bar{h}}}{4}}$ by acting on a celestial superstate $\left|z, \bar{z}, h, \bar{h}, \tau^{A}\right\rangle$. Under a Lorentz transformation,

$\eta^{A}\left|z, \bar{z}, h, \bar{h}, \tau^{A}\right\rangle \rightarrow(c z+d)^{-2 h}(\bar{c} \bar{z}+\bar{d})^{-2 h} \eta^{A}\left|z, \bar{z}, h, \bar{h}, \tau^{A}\right\rangle$.

In order to find the weight values that $h, \bar{h}$ localize onto, we look at the action of $\eta^{A}$ (as given above) on the Kronecker delta. This gives the following shifted spin constraints:

$$
h-\bar{h}=1+\frac{1}{2}, \quad h+\bar{h}=\Delta=1+i \lambda,
$$

hence

$$
h=\frac{\Delta+1}{2}+\frac{1}{4}, \quad \bar{h}=\frac{\Delta-1}{2}-\frac{1}{4} .
$$

We see that the operator $\eta^{A}$ has shifted the weights by $\left(\frac{1}{4},-\frac{1}{4}\right)$ in line with an object with helicity $\frac{1}{2}$, while identical considerations assigns weights $\left(-\frac{1}{4}, \frac{1}{4}\right)$ to $\partial_{A}=\check{\partial}_{A} e^{\frac{\partial_{h}}{4}-\frac{\partial_{\bar{h}}}{4}}$. 


\section{Spinor Derivative: $\partial_{\alpha}$}

The spinor derivative in the supersymmetric theory is written in momentum space according to (6.2) and transforms under a Lorentz transformation as

$$
\begin{aligned}
M_{\alpha}^{\beta} \partial_{\beta} & =\left(\begin{array}{ll}
a & b \\
c & d
\end{array}\right)_{\alpha}^{\beta} \partial_{\beta} \\
& =\frac{1}{u}\left[\left(\begin{array}{l}
a\left(u \partial_{u}+\frac{1}{2} \tau^{A} \check{\partial}_{A}\right) \\
c\left(u \partial_{u}+\frac{1}{2} \tau^{A} \check{\partial}_{A}\right)
\end{array}\right)_{\alpha}-\left(\begin{array}{c}
a z+b \\
c z+d
\end{array}\right)_{\alpha} \partial\right] .
\end{aligned}
$$

Under the combined Möbius coordinate transformation $\left(u^{\prime}, \bar{u}^{\prime}, z^{\prime}, \bar{z}^{\prime}, \tau^{\prime A}\right)$ we have the following partial derivatives,

$$
\begin{aligned}
\partial_{u} & =(c z+d) \partial_{u^{\prime}}, \partial_{\bar{u}}=(\bar{c} \bar{z}+\bar{d}) \partial_{\bar{u}^{\prime}}, \check{\partial}_{A}=\sqrt{\frac{c z+d}{\bar{c} \bar{z}+\bar{d}}} \check{\partial}_{A}^{\prime}, \\
\partial & =\frac{1}{(c z+d)^{2}} \partial^{\prime}+\frac{c}{c z+d} u^{\prime} \partial_{u^{\prime}}+\frac{c}{2(c z+d)} \tau^{\prime A} \check{\partial}_{A}^{\prime}, \\
\bar{\partial} & =\frac{1}{(\bar{c} \bar{z}+\bar{d})^{2}} \bar{\partial}^{\prime}+\frac{\bar{c}}{\bar{c} \bar{z}+\bar{d}} \bar{u}^{\prime} \partial_{\bar{u}^{\prime}}-\frac{\bar{c}}{2(\bar{c} \bar{z}+\bar{d})} \tau^{\prime A} \check{\partial}_{A}^{\prime} .
\end{aligned}
$$

Using (A5) we can write the Lorentz transformed spinor derivative after a few steps as,

$M_{\alpha}^{\beta} \partial_{\beta}=\frac{1}{u^{\prime}}\left[\left(\begin{array}{c}\left(u^{\prime} \partial_{u^{\prime}}+\frac{1}{2} \tau^{\prime A} \check{\partial}_{A}^{\prime}\right) \\ 0\end{array}\right)_{\alpha}-\left(\begin{array}{c}z^{\prime} \\ 1\end{array}\right)_{\alpha} \partial^{\prime}\right]$,

and, as was the case for the chiral spinors in (2.16), we have no need for additional phase factors.

Following Sec. IV B, we can find the weights of the spinor derivative $\partial_{\alpha}$ in Mellin space by acting on a superstate,

$$
\begin{aligned}
& \partial_{\alpha}\left|z, \bar{z}, h, \bar{h}, \tau^{A}\right\rangle \\
& =\int_{\mathbb{C}} d u \wedge d \bar{u} u^{2 h-1} \bar{u}^{2 \bar{h}-1} \frac{1}{u}\left[\left(\begin{array}{c}
u \partial_{u}+\frac{1}{2} \tau^{A} \check{\partial}_{A} \\
0
\end{array}\right)_{\alpha}\right. \\
& \left.\quad-\left(\begin{array}{c}
z \\
1
\end{array}\right)_{\alpha} \partial\right]\left|z^{\prime}, \bar{z}^{\prime}, u^{\prime}, \bar{u}^{\prime}, \tau^{\prime A}\right\rangle,
\end{aligned}
$$

and using (A6) we conclude that this transforms under Lorentz with weight factors equal to $(c z+d)^{-2 h} \times$ $(\bar{c} \bar{z}+\bar{d})^{-2 \bar{h}}$. However, the Kronecker delta appearing in $\partial_{\alpha} \tilde{\mathcal{A}}$ has a shifted spin constraint and the state has a reduced conformal dimension due to the action of $e^{-\partial_{h} / 2}$. Hence, $h-\bar{h}=1+\frac{1}{2}, \quad h+\bar{h}-\frac{1}{2}=\Delta-\frac{1}{2}=1+i \lambda-\frac{1}{2}$,

implying,

$$
h=\frac{\Delta+1}{2}+\frac{1}{4}, \quad \bar{h}=\frac{\Delta-1}{2}-\frac{1}{4} .
$$

Thus the operator $\partial_{\alpha}$ has shifted the weights by $\left(\frac{1}{4},-\frac{1}{4}\right)$ and we assign it weights accordingly and in line with an object with helicity $+\frac{1}{2}$.

\section{Exponential Shift Operator: $e^{\frac{\partial_{h}}{2}}$}

We can also break up generators into their constituent differential operators and study the separate action of those on celestial amplitudes and derive their weights. As an example, consider the action of the exponential operator $e^{\frac{\partial_{h}}{2}}$ on a state. It shifts $(h, \bar{h}) \rightarrow\left(h^{\prime}, \bar{h}^{\prime}\right)$, where we have $h^{\prime}=$ $h+\frac{1}{2}$ while $\bar{h}^{\prime}=\bar{h}$ is left unchanged. Hence,

$$
\begin{aligned}
e^{\frac{\partial_{h}}{2}}|z, \bar{z}, h, \bar{h} ; J\rangle & =\left|z, \bar{z}, h^{\prime}, \bar{h}^{\prime} ; J\right\rangle \\
& =\delta_{h^{\prime}-\bar{h}^{\prime}-J, 0}\left|z, \bar{z}, \Delta^{\prime} ; J\right\rangle,
\end{aligned}
$$

where the conformal dimension has been shifted to $\Delta^{\prime}=$ $\Delta+\frac{1}{2}$ from the conformal dimension of the original state, $\Delta=1+i \beta$. Hence, the weights satisfy

$$
h^{\prime}-\bar{h}^{\prime}=J, \quad h^{\prime}+\bar{h}^{\prime}=\Delta+\frac{1}{2},
$$

which implies

$$
h^{\prime}=\frac{\Delta+J}{2}+\frac{1}{4}, \quad \bar{h}^{\prime}=\frac{\Delta-J}{2}+\frac{1}{4} .
$$

Hence, under a Lorentz transformation

$$
\begin{aligned}
& e^{\frac{\partial_{h}}{2}}|z, \bar{z}, h, \bar{h} ; J\rangle \\
& \rightarrow(c z+d)^{-2 h^{\prime}}(\bar{c} \bar{z}+\bar{d})^{-2 \bar{h}^{\prime}} e^{\frac{\partial_{h}}{2}}\left|z^{\prime}, \bar{z}^{\prime}, h, \bar{h} ; J\right\rangle
\end{aligned}
$$

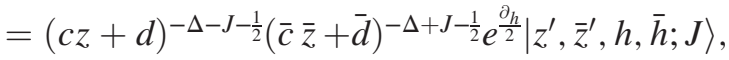

where in the last line we have used (A12). The quantity in (A13) transforms with weights $\left(\frac{\Delta+J}{2}+\frac{1}{4}, \frac{\Delta-J}{2}+\frac{1}{4}\right)$, and compared to the weights $\left(\frac{\Delta+J}{2}, \frac{\Delta-J}{2}\right)$ of the original state we see that the operator $e^{\frac{\partial_{h}}{2}}$ has shifted them by $\left(\frac{1}{4}, \frac{1}{4}\right)$. Hence we conclude that this operator has weights $e^{\frac{\partial_{h}}{2}} \sim\left(\frac{1}{4}, \frac{1}{4}\right)$. We stress that the operator $e^{\frac{\partial_{h}}{2}}$ receives these weights despite shifting $h \rightarrow h^{\prime}=h+\frac{1}{2}$; this is because the spin constraint still requires that $h^{\prime}-\bar{h}^{\prime}=J$ and therefore the new weights $h^{\prime}, \bar{h}^{\prime}$ must correspond to a spin- $J$ object. In other words, the conformal dimension $\Delta$ is the only free parameter of a celestial amplitude and so only it is shifted. 
[1] J. M. Drummond, J. Henn, G. P. Korchemsky, and E. Sokatchev, Dual superconformal symmetry of scattering amplitudes in $N=4$ super-Yang-Mills theory, Nucl. Phys. B828 (2010) 317.

[2] A. Brandhuber, P. Heslop, and G. Travaglini, A note on dual superconformal symmetry of the $N=4$ super Yang-Mills S-matrix, Phys. Rev. D 78 (2008) 125005.

[3] C. Anastasiou, Z. Bern, L. J. Dixon, and D. A. Kosower, Planar Amplitudes in Maximally Supersymmetric YangMills Theory, Phys. Rev. Lett. 91 (2003) 251602.

[4] Z. Bern, L. J. Dixon, and V. A. Smirnov, Iteration of planar amplitudes in maximally supersymmetric Yang-Mills theory at three loops and beyond, Phys. Rev. D 72 (2005) 085001.

[5] J. M. Drummond, J. Henn, V. A. Smirnov, and E. Sokatchev, Magic identities for conformal four-point integrals, J. High Energy Phys. 01 (2007) 064.

[6] L. F. Alday and J. M. Maldacena, Gluon scattering amplitudes at strong coupling, J. High Energy Phys. 06 (2007) 064.

[7] J. M. Drummond, G. P. Korchemsky, and E. Sokatchev, Conformal properties of four-gluon planar amplitudes and Wilson loops, Nucl. Phys. B795 (2008) 385.

[8] A. Brandhuber, P. Heslop, and G. Travaglini, MHV amplitudes in $N=4$ super Yang-Mills and Wilson loops, Nucl. Phys. B794 (2008) 231.

[9] J. M. Drummond, J. Henn, G. P. Korchemsky, and E. Sokatchev, On planar gluon amplitudes/Wilson loops duality, Nucl. Phys. B795 (2008) 52.

[10] S. Pasterski, S.-H. Shao, and A. Strominger, Flat space amplitudes and conformal symmetry of the celestial sphere, Phys. Rev. D 96 (2017) 065026.

[11] S. Pasterski and S.-H. Shao, Conformal basis for flat space amplitudes, Phys. Rev. D 96 (2017) 065022.

[12] S. Pasterski, S.-H. Shao, and A. Strominger, Gluon amplitudes as 2d conformal correlators, Phys. Rev. D 96 (2017) 085006.

[13] J. Terrell, Invisibility of the Lorentz contraction, Phys. Rev. 116 (1959) 1041.

[14] R. Penrose, The Apparent shape of a relativistically moving sphere, Proc. Cambridge Philos. Soc. 55 (1959) 137.

[15] A. Held, E. T. Newman, and R. Posadas, The Lorentz group and the sphere, J. Math. Phys. (N.Y.) 11 (1970) 3145.

[16] B. Oblak, From the Lorentz group to the celestial sphere, arXiv:1508.00920.

[17] V. Lysov, S. Pasterski, and A. Strominger, Low's Subleading Soft Theorem as a Symmetry of QED, Phys. Rev. Lett. 113 (2014) 111601.

[18] C. Cheung, A. de la Fuente, and R. Sundrum, 4D scattering amplitudes and asymptotic symmetries from 2D CFT, J. High Energy Phys. 01 (2017) 112.

[19] L. Donnay, A. Puhm, and A. Strominger, Conformally soft photons and gravitons, J. High Energy Phys. 01 (2019) 184.

[20] E. Himwich and A. Strominger, Celestial current algebra from Low's subleading soft theorem, Phys. Rev. D 100 (2019) 065001.

[21] W. Fan, A. Fotopoulos, and T. R. Taylor, Soft limits of Yang-Mills amplitudes and conformal correlators, J. High Energy Phys. 05 (2019) 121.
[22] M. Pate, A.-M. Raclariu, and A. Strominger, Conformally soft theorem in gauge theory, Phys. Rev. D 100 (2019) 085017.

[23] D. Nandan, A. Schreiber, A. Volovich, and M. Zlotnikov, Celestial amplitudes: Conformal partial waves and soft limits, J. High Energy Phys. 10 (2019) 018.

[24] T. Adamo, L. Mason, and A. Sharma, Celestial amplitudes and conformal soft theorems, Classical Quant. Grav. 36 (2019) 205018.

[25] A. Puhm, Conformally soft theorem in gravity, J. High Energy Phys. 09 (2020) 130.

[26] A. Guevara, Notes on conformal soft theorems and recursion relations in gravity, arXiv:1906.07810.

[27] A. Fotopoulos, S. Stieberger, T. R. Taylor, and B. Zhu, Extended super BMS algebra of celestial CFT, J. High Energy Phys. 09 (2020) 198.

[28] H. Bondi, M. G. J. van der Burg, and A. W. K. Metzner, Gravitational waves in general relativity. 7. Waves from axisymmetric isolated systems, Proc. R. Soc. A 269 (1962) 21.

[29] R. Sachs, Asymptotic symmetries in gravitational theory, Phys. Rev. 128 (1962) 2851.

[30] A. Schreiber, A. Volovich, and M. Zlotnikov, Tree-level gluon amplitudes on the celestial sphere, Phys. Lett. B 781 (2018) 349.

[31] S. Stieberger and T. R. Taylor, Strings on celestial sphere, Nucl. Phys. B935 (2018) 388 .

[32] N. Arkani-Hamed, M. Pate, A.-M. Raclariu, and A. Strominger, Celestial amplitudes from UV to IR, arXiv: 2012.04208.

[33] L. Magnea, Non-abelian infrared divergences on the celestial sphere, J. High Energy Phys. 05 (2021) 282.

[34] H. A. González and F. Rojas, The structure of IR divergences in celestial gluon amplitudes, J. High Energy Phys. 06 (2021) 171.

[35] E. Himwich, S. A. Narayanan, M. Pate, N. Paul, and A. Strominger, The soft $\mathcal{S}$-matrix in gravity, J. High Energy Phys. 09 (2020) 129.

[36] S. Albayrak, C. Chowdhury, and S. Kharel, On loop celestial amplitudes for gauge theory and gravity, Phys. Rev. D 102 (2020) 126020.

[37] H. A. González, A. Puhm, and F. Rojas, Loop corrections to celestial amplitudes, Phys. Rev. D 102 (2020) 126027.

[38] S. Pasterski, A. Puhm, and E. Trevisani, Celestial diamonds: Conformal multiplets in celestial CFT, arXiv:2105.03516.

[39] S. Stieberger and T. R. Taylor, Symmetries of celestial amplitudes, Phys. Lett. B 793 (2019) 141.

[40] N. Kalyanapuram, Gauge and gravity amplitudes on the celestial sphere, Phys. Rev. D 103 (2021) 085015.

[41] J. M. Drummond, J. M. Henn, and J. Plefka, Yangian symmetry of scattering amplitudes in $N=4$ super YangMills theory, J. High Energy Phys. 05 (2009) 046.

[42] V.P. Nair, A current algebra for some gauge theory amplitudes, Phys. Lett. B 214 (1988) 215.

[43] E. Witten, Perturbative gauge theory as a string theory in twistor space, Commun. Math. Phys. 252 (2004) 189.

[44] H. Jiang, Celestial superamplitude in $\mathcal{N}=4$ SYM theory, arXiv:2105.10269. 